\title{
Continuous unitary transformations and finite-size scaling exponents in the Lipkin-Meshkov-Glick model
}

\author{
Sébastien Dusuel ${ }^{1, \circledast}$ and Julien Vidal ${ }^{2, \text { 团 }}$ \\ ${ }^{1}$ Institut für Theoretische Physik, Universität zu Köln, Zülpicher Str. 77, 50937 Köln, Germany \\ ${ }^{2}$ Laboratoire de Physique Théorique de la Matière Condensée, CNRS UMR 7600, \\ Université Pierre et Marie Curie, 4 Place Jussieu, 75252 Paris Cedex 05, France
}

\begin{abstract}
We analyze the finite-size scaling exponents in the Lipkin-Meshkov-Glick model by means of the Holstein-Primakoff representation of the spin operators and the continuous unitary transformations method. This combination allows us to exactly compute the leading corrections to the groundstate energy, the gap, the magnetization, and the two-spin correlation functions. We also present numerical calculations for large system size which confirm the validity of this approach. Finally, we use these results to discuss the entanglement properties of the ground state focusing on the (rescaled) concurrence that we compute in the thermodynamical limit.
\end{abstract}

PACS numbers: 75.40.Cx,05.10.Cc,11.10.Hi,03.65.Ud

\section{INTRODUCTION}

A growing interest has recently been devoted to the study of quantum phase transitions in spin systems especially from the entanglement point of view. The ground-state intricated structure has indeed been shown to be strongly affected by the existence of a critical point as initially illustrated in the one-dimensional $(1 D)$ Ising model under magnetic field ${ }^{1.2}$. Following these pioneering works, many systems have been studied and have revealed a specific behavior of the entanglement as measured either by

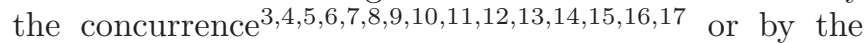
entropy 18.19.20.21.22.23.24. In this context, the LipkinMeshkov-Glick (LMG) model25.26.27 has focused much attention because of its apparent simplicity. Introduced fourty years ago in nuclear physics, this model provides a simple description of the tunneling of bosons between two degenerate levels and can thus be used to describe many physical systems such as two-mode Bose-Einstein condensates ${ }^{28}$ or Josephson junctions. In the thermodynamical limit, its phase diagram can be simply established using a semiclassical (mean-field) approach ${ }^{29.30}$. However, at finite size, the situation is more complicated and crucial to understand the entanglement properties of

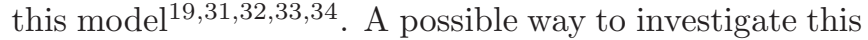
problem is to use the exact solution provided by the algebraic Bethe ansatz ${ }^{35.36}$, but it would require the computation of the correlation functions which is a rather difficult task with this formalism.

In the present work, we present an alternative route which relies on a combination of two well-known methods, and which we have already briefly sketched in Ref. 34. First, we use the Holstein-Primakoff representation which allows us to write a $1 / N$ expansion of the spin operators at arbitrary order, $N$ being the number of degrees of freedom. Usually, such a development is often restricted to the first order for which the Hamiltonian remains quadratic and can thus be easily diagonalized. We show that this first order calculation does not provide us with enough information. This implies one has to take more terms in the $1 / N$ expansion, and thus to deal with a nonquadratic Hamiltonian. Here, we use the continuous unitary transformations (CUTs) technique proposed by Wegner ${ }^{37}$ and independently by Głazek and Wilson ${ }^{38.39}$, to compute the next orders. The structure of this expansion contains all relevant informations to extract the finite size corrections of various observables at the critical point. We consider in particular the ground-state energy, the gap, the magnetization, and the spin-spin correlation function for which we obtain nontrivial scaling exponents. These results corroborate several numerical studies 15,30 in which some of these exponents have already been computed.

In addition to giving details of the calculations yielding the results announced in Ref. 34, we present a numerical study for all the finite-size scaling exponents which corroborates the analytical predictions. Furthermore, we investigate the broken phase whereas in Ref. 34, we only considered the symmetric one. Finally, we compute the leading order two-spin entanglement in the thermodynamical limit for any anisotropy parameter.

This paper is organized as follows. In Sec. III we introduce the LMG model, discuss its symmetries and give its simple solution for the isotropic case. In Sec. III] we recall the variational approach which constitutes the zeroth order of the $1 / N$ expansion but which is sufficient to establish the phase diagram in the thermodynamical limit. Section $\mathbb{\mathbb { }}$ is devoted to the first order corrections that are easily computed via the standard Bogoliubov transformation. In the next section (Sec. V) we introduce the basics of the CUT formalism and apply it to the LMG Hamiltonian. These corrections allow us to give analytical expressions of the finite-size scaling exponents. In Sec. VI] we present a numerical study that confirms our results and sheds light on the discrepancy with previous numerics. Then, in Sec. VII we discuss the entanglement properties of the ground state focusing on the so-called concurrence which measures the two-spin entanglement. We show, in particular, that it displays a 
cusp at the transition point contrary to what is observed in $1 D$ systems. Technical details are given in Appendix.

\section{THE LIPKIN-MESHKOV-GLICK MODEL}

\section{A. The Hamiltonian and its symmetries}

The LMG model25.26.27 describes a set of $N$ spins half mutually interacting in the (anisotropic) $x-y$ plane embedded in a perpendicular magnetic field pointing in the $z$ direction. The corresponding Hamiltonian is written

$$
\begin{aligned}
H= & -\frac{\lambda}{N} \sum_{i<j}\left(\sigma_{x}^{i} \sigma_{x}^{j}+\gamma \sigma_{y}^{i} \sigma_{y}^{j}\right)-h \sum_{i} \sigma_{z}^{i}, \\
= & -\frac{2 \lambda}{N}\left(S_{x}^{2}+\gamma S_{y}^{2}\right)-2 h S_{z}+\frac{\lambda}{2}(1+\gamma), \\
= & -\frac{\lambda}{N}(1+\gamma)\left(\mathbf{S}^{2}-S_{z}^{2}-N / 2\right)-2 h S_{z} \\
& -\frac{\lambda}{2 N}(1-\gamma)\left(S_{+}^{2}+S_{-}^{2}\right),
\end{aligned}
$$

where the $\sigma_{\alpha}$ 's are the Pauli matrices, $S_{\alpha}=\sum_{i} \sigma_{\alpha}^{i} / 2$, and $S_{ \pm}=S_{x} \pm \mathrm{i} S_{y}$. The prefactor $1 / N$ is necessary to obtain a finite free energy per spin in the thermodynamical limit. For any anisotropy parameter $\gamma, H$ preserves the magnitude of the total spin and does not couple states having a different number of spins pointing in the field direction (spin-flip symmetry), namely

$$
\left[H, \mathbf{S}^{2}\right]=0 \quad \text { and } \quad\left[H, \prod_{i} \sigma_{z}^{i}\right]=0 .
$$

An important consequence of the spin-flip symmetry is that any eigenstates of $\prod_{i} \sigma_{z}^{i}$ satisfy

$$
\left\langle S_{x}\right\rangle=\left\langle S_{y}\right\rangle=0
$$

and

$$
\begin{aligned}
& \left\langle S_{x} S_{z}\right\rangle=\left\langle S_{z} S_{x}\right\rangle=0 \\
& \left\langle S_{y} S_{z}\right\rangle=\left\langle S_{z} S_{y}\right\rangle=0 .
\end{aligned}
$$

In the following, we only consider a ferromagnetic dominant coupling $(\lambda>0,|\gamma| \leq 1)$ and without loss of generality, we set $\lambda=1$. For a discussion of the antiferromagnetic case, we refer the reader to Ref. 32. We also restrict our discussion to the region $h \geq 0$ since the spectrum is even under the transformation $h \leftrightarrow-h$. In addition, we only consider the maximum spin sector $S=N / 2$ which contains the low-energy states.

The ground-state properties can be easily studied in the thermodynamical limit using a mean-field analysis that we briefly recall in the following section. It is worth however to first have a look at the isotropic model for which a complete analytical solution is available.

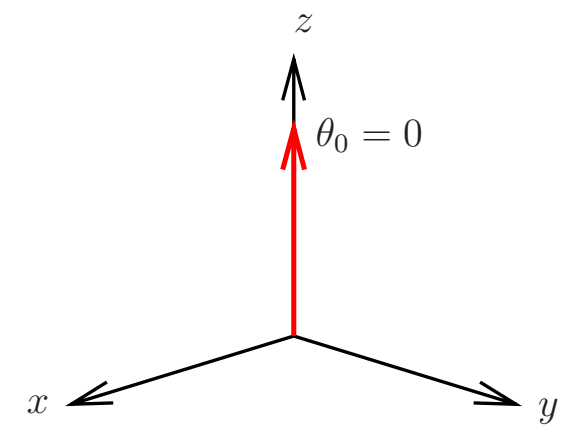

FIG. 1: The mean-field ground state in the symmetric phase, for all values of the anisotropy parameter $\gamma$.

\section{B. Analytical solution of the isotropic model}

In the isotropic case $\gamma=1$, the Hamiltonian (3) reads

$$
H=-\frac{2}{N}\left(\mathbf{S}^{2}-S_{z}^{2}-\frac{N}{2}\right)-2 h S_{z} .
$$

$H$ thus commutes both with $\mathbf{S}^{2}$ and $S_{z}$, so that it is diagonal in the standard eigenbasis $\{|S, M\rangle\}$ of $\mathbf{S}^{2}$ and $S_{z}$. The eigenenergies are

$$
E(S, M)=-\frac{2}{N}\left[S(S+1)-M^{2}-N / 2\right]-2 h M
$$

For the ferromagnetic case that we consider here, the ground state is then obtained for $S=N / 2$ and

$$
M_{0}=\left\{\begin{array}{clc}
I(h N / 2) & \text { for } & 0 \leq h<1 \\
N / 2 & \text { for } & h \geq 1
\end{array},\right.
$$

where $I(x)$ gives the integer part of $x$, in the following sense : if $x=X+\delta x$ with $X$ an integer and $\delta x \in[0,1[$, then $I(x)=X$ for $\delta x \in[0,1 / 2[$ and $I(x)=X+1$ for $\delta x \in[1 / 2,1[$. One then trivially gets

$$
\begin{aligned}
& e_{0}(N)=-\frac{1}{2}+\frac{2 M_{0}^{2}}{N^{2}}-\frac{2 h M_{0}}{N} \\
& \frac{2\left\langle S_{x}\right\rangle}{N}=0 \\
& \frac{2\left\langle S_{y}\right\rangle}{N}=0 \\
& \frac{2\left\langle S_{z}\right\rangle}{N}=\frac{2 M_{0}}{N}, \\
& \frac{4\left\langle S_{x}^{2}\right\rangle}{N^{2}}=\frac{4\left\langle S_{y}^{2}\right\rangle}{N^{2}}=\frac{2}{N^{2}}\left[\frac{N}{2}\left(\frac{N}{2}+1\right)-M_{0}^{2}\right] \\
& \frac{4\left\langle S_{z}^{2}\right\rangle}{N^{2}}=\frac{4 M_{0}^{2}}{N^{2}} .
\end{aligned}
$$

where $e_{0}(N)$ is the ground-state energy per spin. The gap can also be easily computed :

$$
\begin{aligned}
\Delta(N) & =E\left(N / 2, M_{0}+s\right)-E\left(N / 2, M_{0}\right) \\
& =\frac{2}{N}+2 s\left(2 M_{0} / N-h\right),
\end{aligned}
$$




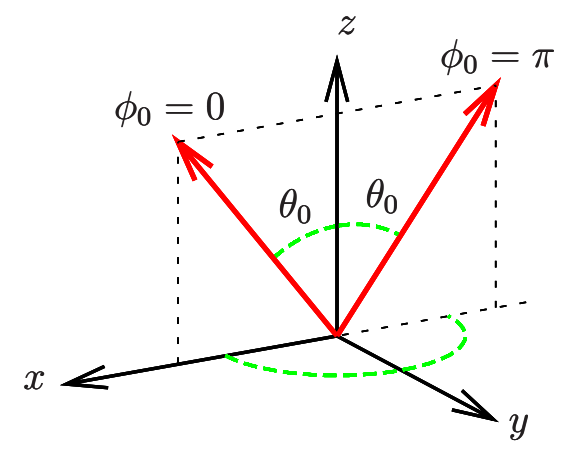

FIG. 2: The two mean-field ground states in the broken phase of the anisotropic LMG model $\gamma<1$.

where $s=\operatorname{sgn}\left(h N / 2-M_{0}\right)$ if $0<h<1$ and $s=-1$ if $h \geq 1$. This gives $\Delta(N)=2 / N+2 s\left(2 M_{0} / N-h\right)$ in the broken phase [which is a $2 / N$-periodic function vanishing for $h=(2 p+1) / N$ and equal to $2 / N$ for $h=2 p / N, p$ denoting an integer], whereas $\Delta(N)=2(h-1)+2 / N$ in the symmetric phase.

\section{VARIATIONAL AND SEMICLASSICAL APPROACHES}

In this section, we describe the semiclassical approach that can be used to determined the phase diagram of the LMG model ${ }^{29.30}$ This analysis constitutes the zeroth order approximation and relies on a mean-field (variational) wave function

$|\psi(\theta, \phi)\rangle=\bigotimes_{l=1}^{N}\left[\cos (\theta / 2) e^{-\mathrm{i} \phi / 2}|\uparrow\rangle_{l}+\sin (\theta / 2) e^{\mathrm{i} \phi / 2}|\downarrow\rangle_{l}\right]$,

which is a coherent spin state such that

$$
\langle\mathbf{S}\rangle=\frac{N}{2}(\sin \theta \cos \phi, \sin \theta \sin \phi, \cos \theta) .
$$

In Eq. (19), kets $|\uparrow\rangle_{l}$ and $|\downarrow\rangle_{l}$ are the eigenstates of $\sigma_{z}^{l}$ with eigenvalues +1 and -1 , respectively. The ground state is thus determined by minimizing the energy

$$
\langle H\rangle=-\frac{(N-1)}{2} \sin ^{2} \theta\left(\cos ^{2} \phi+\gamma \sin ^{2} \phi\right)-h N \cos \theta .
$$

with respect to $\theta$ and $\phi$.

In the thermodynamical limit, this variational approach is completely equivalent to the semiclassical treatment proposed by Botet and Jullien ${ }^{30}$ which consists in treating $\mathbf{S}$ as a classical variable. In this limit, minimizing $\langle H\rangle$ with respect to $\theta$ and $\phi$ leads to a distinction between the two phases

1. $h \geq 1$ (symmetric phase): The ground state is unique and fully polarized in the magnetic field direction $\left(\theta_{0}=0\right)$ for all $\gamma$, see Fig. 1

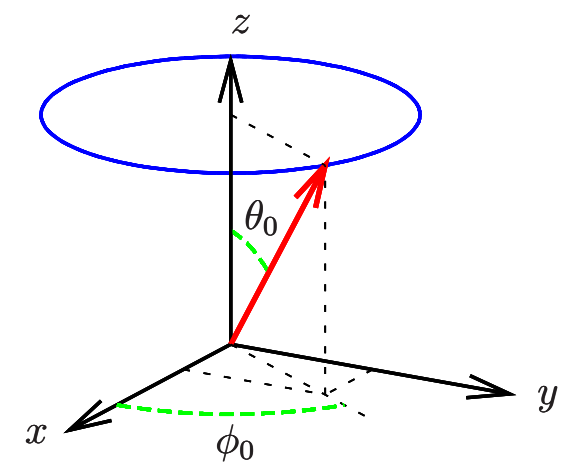

FIG. 3: One of the infinitely degenerate mean-field ground states in the broken phase of the isotropic LMG model $\gamma=1$.

\section{2. $0 \leq h<1$ (broken phase):}

For $\gamma \neq 1$, the ground state is twofold degenerate $\left(\theta_{0}=\arccos h\right.$ and $\phi_{0}=0$ or $\pi$ ) (see Fig. 2). In the isotropic case $\gamma=1,\langle H\rangle$ does not depend on $\phi$ so that the ground state is infinitely degenerate, as shown in Fig. 3. Of course, this is due to the parametrization of the trial function. However, if this result is clearly in contradiction with the exact degeneracy computed in Sec. IIB (which is unique at least for $h \neq 0$ ), it points out the existence of two universality classes $(\gamma=1$ and $\gamma \neq 1)$.

Here, $\theta_{0}$ and $\phi_{0}$ stands for the values of $\theta$ and $\phi$ for which $\langle H\rangle$ is minimum. The ground-state energy per spin is then given by:

$$
e_{0}(\infty)=-\frac{1}{2} \sin ^{2} \theta_{0}-h \cos \theta_{0}
$$

and the gap $\Delta$ can be evaluated using the random phase approximation $^{30}$

$$
\Delta(\infty)=\left\{\begin{array}{cl}
2[(h-1)(h-\gamma)]^{1 / 2} & \text { for } \quad h \geq 1, \\
0 & \text { for } 0 \leq h<1 .
\end{array}\right.
$$

Let us note that, for the anisotropic LMG model, the result in the broken phase $0 \leq h<1$ simply says that there are two degenerate ground states, and is obtained without performing a calculation. This gap does not correspond to the excitation energy around one of the two mean-field states represented in Fig. 2 which is finite. The latter is denoted as $\Delta^{\prime}$ in Fig. 目 whereas in the same figure, $\Delta$ denotes the exact gap, vanishing in the broken phase and in the thermodynamical limit.

The correlation functions are also easily obtained in the thermodynamical limit since, for a "classical" spin one simply has $\left\langle S_{\alpha} S_{\beta}\right\rangle=\left\langle S_{\alpha}\right\rangle\left\langle S_{\beta}\right\rangle+O(N)$. Note that for $\gamma=1$, one can recover the exact expressions derived in $1 \mathrm{BB}$ provided an average over all possible values of $\phi_{0}$ is performed. For example, one has:

$$
\frac{4\left\langle S_{x}^{2}\right\rangle}{N^{2}}=\frac{4\left\langle S_{x}\right\rangle\left\langle S_{x}\right\rangle}{N^{2}}+O(1 / N)
$$




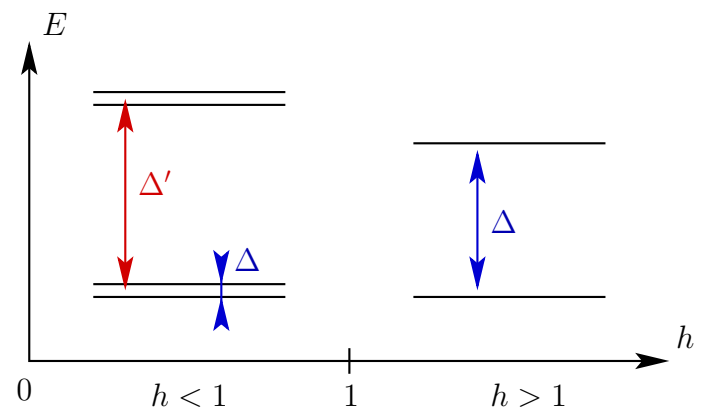

FIG. 4: Schematical representation of the low-energy spectrum of the anisotropic LMG model in a finite system. $\Delta$ is the energy gap between the ground state and the first excited state. In the broken phase $h<1, \Delta$ is exponentially small $\Delta \sim \exp (-a N) \stackrel{40}{*}$ In this phase, $\Delta^{\prime}$ is the excitation energy between the two nearly degerate lowest states and the nearly degenerate next two states.

$$
\begin{aligned}
& =\sin ^{2} \theta_{0} \overline{\cos ^{2} \phi_{0}}+O(1 / N), \\
& =\frac{1}{2} \sin ^{2} \theta_{0}+O(1 / N) .
\end{aligned}
$$

which coincides with Eq. (15) in the infinite $N$ limit.

In the thermodynamical limit, this mean-field approximation is exact (see the comparison with the numerical results in Sec. VI and thus predicts a second-order quantum phase transition at the critical field $h=1$.

For finite $N$, the exact ground state is nondegenerate and is not given by a mean-field state $|\psi(\theta, \phi)\rangle$ except for $h=\frac{N-1}{N} \sqrt{\gamma}$. The twofold degeneracy (for $\gamma \neq 1$ ) in the broken phase only occurs in the infinite $N$ limit where the two mean-field solutions $\left|\psi\left(\theta_{0}, 0\right)\right\rangle$ and $\left|\psi\left(\theta_{0}, \pi\right)\right\rangle$ provides a (nonorthogonal) basis of the ground-state subspace.

Finally, from the entanglement point of view, $\left|\psi\left(\theta_{0}, \phi_{0}\right)\right\rangle$ is a completely separable state but as discussed in Sec. VII the exact ground state has some nontrivial intrication properties (even in the thermodynamical limit) that cannot be captured at this level. It is thus essential to go beyond this basic approach and to compute next orders in the $1 / N$ expansion.

\section{FIRST ORDER QUANTUM CORRECTIONS}

We now wish to go one step beyond the mean-field analysis of the previous section by computing first order correction in a $1 / N$ expansion. To achieve this, we use the Holstein-Primakoff representation of the spin operator and truncate the resulting bosonic Hamiltonian to lowest order. Next, we diagonalize it thanks to a Bogoliubov transformation.

\section{A. The Holstein-Primakoff representation}

The Holstein-Primakoff representation of spin operators 41 is a useful tool to compute low-energy corrections around a classical magnetization (see for example Ref. 42). The very first thing to do is thus to perform a rotation of the spin operators around the $y$ axis, that brings the $z$ axis along the semiclassical magnetization. This is done as follows

$$
\left(\begin{array}{c}
S_{x} \\
S_{y} \\
S_{z}
\end{array}\right)=\left(\begin{array}{ccc}
\cos \theta_{0} & 0 & \sin \theta_{0} \\
0 & 1 & 0 \\
-\sin \theta_{0} & 0 & \cos \theta_{0}
\end{array}\right)\left(\begin{array}{c}
\tilde{S}_{x} \\
\tilde{S}_{y} \\
\tilde{S}_{z}
\end{array}\right) .
$$

As explained in the previous section, $\theta_{0}=0$ for $h>1$ so that $\mathbf{S}=\tilde{\mathbf{S}}$, and $\theta_{0}=\arccos h$ for $h \leq 1$. Note that we have chosen here the magnetization direction corresponding to $\phi_{0}=0$ but the same results can be obtained by choosing $\phi_{0}=\pi$.

The Hamiltonian (3) written in terms of the $\tilde{\mathbf{S}}$ then reads

$$
\begin{aligned}
H= & \frac{1+\gamma}{2}-2 h m \tilde{S}_{z} \\
& -\frac{2}{N}\left[\left(1-m^{2}\right) \tilde{S}_{z}^{2}+\frac{m^{2}+\gamma}{2}\left(\tilde{\mathbf{S}}^{2}-\tilde{S}_{z}^{2}\right)\right] \\
& +h \sqrt{1-m^{2}}\left(\tilde{S}_{+}+\tilde{S}_{-}\right) \\
& -\frac{m \sqrt{1-m^{2}}}{N}\left(\tilde{S}_{+} \tilde{S}_{z}+\tilde{S}_{z} \tilde{S}_{+}+\tilde{S}_{-} \tilde{S}_{z}+\tilde{S}_{z} \tilde{S}_{-}\right) \\
& -\frac{m^{2}-\gamma}{2 N}\left(\tilde{S}_{+}^{2}+\tilde{S}_{-}^{2}\right)
\end{aligned}
$$

where $m=2\left\langle S_{z}\right\rangle / N=\cos \theta_{0}$. The Holstein-Primakoff representation is then applied to the rotated spin operators

$$
\begin{aligned}
& \tilde{S}_{z}=S-a^{\dagger} a=N / 2-a^{\dagger} a, \\
& \tilde{S}_{+}=\left(2 S-a^{\dagger} a\right)^{1 / 2} a=N^{1 / 2}\left(1-a^{\dagger} a / N\right)^{1 / 2} a, \\
& \tilde{S}_{-}=a^{\dagger}\left(2 S-a^{\dagger} a\right)^{1 / 2}=N^{1 / 2} a^{\dagger}\left(1-a^{\dagger} a / N\right)^{1 / 2},
\end{aligned}
$$

where the bosonic creation and annihilation operators satisfy $\left[a, a^{\dagger}\right]=1$.

The next step consists in inserting these expressions in Eq. (28), and to expand the argument of the square roots. Keeping terms of order $(1 / N)^{-1},(1 / N)^{-1 / 2}$ and $(1 / N)^{0}$ in the Hamiltonian [therefore, it is sufficient to use the approximation $\left.\left(1-a^{\dagger} a / N\right)^{1 / 2} \simeq 1\right]$ yields

$$
H^{(0)}=N e_{0}(\infty)+\delta e_{0}^{(\mathrm{B})}+\Delta^{(\mathrm{B})} a^{\dagger} a+\Gamma^{(\mathrm{B})}\left(a^{\dagger^{2}}+a^{2}\right),
$$

where $e_{0}(\infty)$ is the mean-field ground-state energy per spin (22), which also reads $e_{0}(\infty)=\left(-1-2 h m+m^{2}\right) / 2$. The ${ }^{(B)}$ superscript stands for "bare", and the bare couplings are

$$
\begin{aligned}
\delta e_{0}^{(\mathrm{B})} & =\frac{1-m^{2}}{2}, \\
\Delta^{(\mathrm{B})} & =2+2 h m-3 m^{2}-\gamma, \\
\Gamma^{(\mathrm{B})} & =\frac{\gamma-m^{2}}{2} .
\end{aligned}
$$


We emphasize that the Hamiltonian contains no term proportional to $\sqrt{N}\left(a^{\dagger}+a\right)$, i.e., of order $(1 / N)^{-1 / 2}$, creating one excitation. These terms simply cancels because the angle of the rotation (27) has been chosen to bring $\tilde{S}_{z}$ along the classical magnetization. Note that this can also be achieved, as in Ref. 43, for example, by applying directly the Holstein-Primakoff mapping to the nonrotated $\mathbf{S}$ operator, and performing a shift of $a^{\dagger}$ and $a$ by an appropriate constant.

\section{B. The Bogoliubov transformation}

The quadratic Hamiltonian (32) is easily diagonalized by a standard Bogoliubov transformation

$$
\begin{aligned}
a^{\dagger} & =\cosh (\Theta / 2) b^{\dagger}+\sinh (\Theta / 2) b, \\
a & =\sinh (\Theta / 2) b^{\dagger}+\cosh (\Theta / 2) b .
\end{aligned}
$$

where $\Theta$ is such that the Hamiltonian expressed in terms of the $b$ 's does not contain a term $b^{\dagger^{2}}+b^{2}$, i.e. is diagonal. Let us set $\varepsilon=-2 \Gamma^{(\mathrm{B})} / \Delta^{(\mathrm{B})}$, which takes the following values in the symmetric and broken phases

$$
\begin{aligned}
\varepsilon(h \geq 1) & =\frac{1-\gamma}{2 h-1-\gamma}, \\
\varepsilon(0 \leq h<1) & =\frac{h^{2}-\gamma}{2-h^{2}-\gamma} .
\end{aligned}
$$

One easily finds that to diagonalize the Hamiltonian (32) one must satisfy $\tanh \Theta=\varepsilon$. One then has

$$
H^{(0)}=N e_{0}(\infty)+\delta e_{0}^{(\mathrm{R})}+\Delta^{(\mathrm{R})} b^{\dagger} b,
$$

where the ${ }^{(\mathrm{R})}$ superscript means "renormalized" and the renormalized couplings are given by

$$
\begin{aligned}
\delta e_{0}^{(\mathrm{R})} & =\delta e_{0}^{(\mathrm{B})}+\frac{\Delta^{(\mathrm{B})}}{2}\left(\sqrt{1-\varepsilon^{2}}-1\right), \\
\Delta^{(\mathrm{R})} & =\Delta^{(\mathrm{B})} \sqrt{1-\varepsilon^{2}} .
\end{aligned}
$$

At this stage, it is worth noting that, in the broken phase, for $h=\sqrt{\gamma}, \varepsilon$ and thus $\Gamma^{(\mathrm{B})}$ vanishes so that the Hamiltonian (32) is readily diagonal. This special point coincides, at this order $(1 / N)^{0}$, with $h=\frac{N-1}{N} \sqrt{\gamma}$ previously mentioned (see Sec. III) for which the product state $\left|\psi\left(\theta_{0}, \phi_{0}\right)\right\rangle$ is the exact ground state.

In the symmetric phase $m=1$, one thus gets

$$
\begin{aligned}
\delta e_{0}^{(\mathrm{R})} & =-h+\frac{1+\gamma}{2}+[(h-1)(h-\gamma)]^{1 / 2}, \\
\Delta^{(\mathrm{R})} & =2[(h-1)(h-\gamma)]^{1 / 2} .
\end{aligned}
$$

The last equation thus gives the gap (23) found in the random phase approximation.

In the broken phase $m=h$ and

$$
\begin{aligned}
\delta e_{0}^{(\mathrm{R})} & =-\frac{1-\gamma}{2}+\left[\left(1-h^{2}\right)(1-\gamma)\right]^{1 / 2}, \\
\Delta^{(\mathrm{R})} & =2\left[\left(1-h^{2}\right)(1-\gamma)\right]^{1 / 2} .
\end{aligned}
$$

In this case, one must be careful about the physical interpretation of $\Delta^{(R)}$. It is not the true gap of the system, opened by the tunneling between the two classical ground states, which is known ${ }^{40}$ to be exponentially small $[\sim \exp (-a N)]$. It is thus not the quantity denoted as $\Delta$ in Fig. 4 but the one denoted as $\Delta^{\prime}$, which is the excitation energy in the vicinity of one of the classical magnetization [up to some terms of order $\exp (-a N)$ which are not accessible in the $1 / N$ expansion]. It is thus obtained by computing the energy difference between the ground state and the second excited state. For the same reasons, the ground-state energy as given by $\delta e_{0}^{(\mathrm{R})}$ is only valid up to exponentially small terms.

Finally, we can compute the first quantum correction to the observables by using Eq. (27) and the expressions of the $\tilde{\mathbf{S}}$ operator truncated to lowest order and expressed with the $b$ 's

$$
\begin{aligned}
& \tilde{S}_{x} \simeq \frac{N^{1 / 2}}{2}\left(a^{\dagger}+a\right) \\
&=\frac{N^{1 / 2}}{2}\left(\frac{1+\varepsilon}{1-\varepsilon}\right)^{1 / 4}\left(b^{\dagger}+b\right) \\
& \tilde{S}_{y} \simeq \frac{\mathrm{i} N^{1 / 2}}{2}\left(a^{\dagger}-a\right) \\
&=\frac{\mathrm{i} N^{1 / 2}}{2}\left(\frac{1-\varepsilon}{1+\varepsilon}\right)^{1 / 4}\left(b^{\dagger}-b\right), \\
& \tilde{S}_{z}=\frac{N}{2}+\frac{1}{2}\left(1-\frac{1}{\sqrt{1-\varepsilon^{2}}}\right)-\frac{1}{\sqrt{1-\varepsilon^{2}}} b^{\dagger} b \\
&-\frac{\varepsilon}{2 \sqrt{1-\varepsilon^{2}}}\left(b^{\dagger^{2}+b^{2}}\right) .
\end{aligned}
$$

The one spin averages in the rotated basis deduced from these formulas read

$$
\begin{aligned}
& 2\left\langle\tilde{S}_{x}\right\rangle / N=2\left\langle\tilde{S}_{y}\right\rangle / N=0+O(1 / N), \\
& 2\left\langle\tilde{S}_{z}\right\rangle / N=1+\frac{1}{N}\left(1-\frac{1}{\sqrt{1-\varepsilon^{2}}}\right)+O\left(1 / N^{2}\right) .
\end{aligned}
$$

In the symmetric phase, these expressions can be used by simply replacing the $\widetilde{S}$ by $S$, and by assigning the expression (38) to $\varepsilon$. To obtain the results in the broken phase, one should pay attention to the fact that the corrections to $\left\langle\tilde{S}_{x}\right\rangle$ and $\left\langle\tilde{S}_{y}\right\rangle$ on the one hand and to $\left\langle\tilde{S}_{z}\right\rangle$ on the other, are not of the same order. One then gets

$$
\begin{aligned}
& 2\left\langle S_{x}\right\rangle / N=\sqrt{1-m^{2}}, \\
& 2\left\langle S_{y}\right\rangle / N=0, \\
& 2\left\langle S_{z}\right\rangle / N=m,
\end{aligned}
$$

which are nothing but the mean-field results. In order to obtain the first quantum correction to these results in the broken phase, one must also be able to compute the $1 / N$ corrections to $2\left\langle\tilde{S}_{x}\right\rangle / N$ and to $2\left\langle\tilde{S}_{y}\right\rangle / N$. Such a calculation requires that we go beyond the Bogoliubov transformation and will be presented in Sec. V It is nonetheless possible to extract some of the first quantum corrections to spin-spin correlation function in both 
phases

$$
\begin{aligned}
4\left\langle\tilde{S}_{x}^{2}\right\rangle / N^{2} & =\frac{1}{N}\left(\frac{1+\varepsilon}{1-\varepsilon}\right)^{1 / 2}, \\
4\left\langle\tilde{S}_{y}^{2}\right\rangle / N^{2} & =\frac{1}{N}\left(\frac{1-\varepsilon}{1+\varepsilon}\right)^{1 / 2}, \\
4\left\langle\tilde{S}_{z}^{2}\right\rangle / N^{2} & =1+\frac{2}{N}\left(1-\frac{1}{\sqrt{1-\varepsilon^{2}}}\right), \\
4\left\langle\tilde{S}_{x} \tilde{S}_{y}\right\rangle / N^{2} & =4\left\langle\tilde{S}_{y} \tilde{S}_{x}\right\rangle^{*} / N^{2}=\frac{\mathrm{i}}{N}, \\
4\left\langle\tilde{S}_{x} \tilde{S}_{z}\right\rangle / N^{2} & =4\left\langle\tilde{S}_{z} \tilde{S}_{x}\right\rangle^{*} / N^{2}=O(1 / N), \\
4\left\langle\tilde{S}_{y} \tilde{S}_{z}\right\rangle / N^{2} & =4\left\langle\tilde{S}_{z} \tilde{S}_{y}\right\rangle^{*} / N^{2}=O(1 / N) .
\end{aligned}
$$

Here again, the Bogoliubov transformation is a too simple calculation to obtain all the $1 / N$ corrections. In the symmetric phase however, we know that the spin-flip symmetry is not broken, so that Eqs. (59) and (60) in fact vanish. In the broken phase, although Eq. (60) still vanishes, Eq. (59) is nonvanishing. We refer the reader to Appendix where we have gathered the first $1 / N$ corrections (computed as explained in the next section). Let us mention that sticking to the results (55)- (60) presently available, the only two-spin correlations we can compute in both phases, and better than in the mean-field treatment is

$$
4\left\langle S_{y}^{2}\right\rangle / N^{2}=4\left\langle\tilde{S}_{y}^{2}\right\rangle / N^{2}=\frac{1}{N}\left(\frac{1-\varepsilon}{1+\varepsilon}\right)^{1 / 2} .
$$

Luckily, as explained in Sec. VIIA this is precisely the correlation function that is required to compute the rescaled concurrence in the parameter regime $h \geq 0$ for $\gamma \leq 0$ or $h \geq \sqrt{\gamma}$ for $\gamma \geq 0$ [see Eq. [105]). However, when $\gamma \geq 0$ and $h \geq \sqrt{\gamma}$, one needs to know the $1 / N$ corrections to $\left\langle S_{z}\right\rangle$ and $\left\langle S_{z}^{2}\right\rangle$ [see Eq. (108)], which can not be computed with the Bogoliubov transform.

Of course, it would be tempting to go beyond these first-order corrections pushing the expansion of the spin operator to the next order. Unfortunately, from the order $(1 / N)^{1 / 2}$, the Hamiltonian is no more quadratic and, consequently, cannot be diagonalized by a Bogoliubov transformation. This clearly calls for a more sophisticated method which constitutes the aim of this article and is presented in the next section.

\section{HIGHER ORDER QUANTUM CORRECTIONS}

In this section, we show how to to go beyond the firstorder quantum corrections given previously. We explain how to compute higher-order corrections in the $1 / N$ expansion thanks to the CUTs technique. These results are used, together with a scaling argument, in order to extract the finite-size scaling exponents of various physical quantities at critical coupling. The analytical calculation of these exponents is the central result of this study.

\section{A. Introduction to continuous unitary transformations}

To compute higher order terms in the $1 / N$ expansion, we need to diagonalize the Hamiltonian (28) truncated at the corresponding order, i.e. to perform a unitary transformation that brings the Hamiltonian to a diagonal form. Finding such an unitary transformation is clearly not an easy task. It was the idea of Wegner ${ }^{37}$ ten years ago, and independently of Głazek and Wilson ${ }^{38.39}$ to replace the single step unitary transformation by an infinite product of infinitesimal transformations, that is by CUTs. In this way, one does not have to solve an algebraic problem but a set of differential equations.

We shall now briefly recall the formalism of the CUTs, and refer the reader to Ref. 44 for a pedagogical introduction and more references on this topic. The idea of the CUTs is to diagonalize the Hamiltonian in a continuous way starting from the original (bare) Hamiltonian $H=H(l=0)$. A flowing Hamiltonian is thus defined by

$$
H(l)=U^{\dagger}(l) H(0) U(l),
$$

where $l$ is a scaling parameter such that $H(l=\infty)$ is diagonal and $U(l)$ is a unitary transformation, i.e., satisfying $U(l) U^{\dagger}(l)=U^{\dagger}(l) U(l)=1$. A derivation of Eq. [62] with respect to $l$ yields the differential equation (called the flow equation)

$$
\partial_{l} H(l)=[\eta(l), H(l)],
$$

where the generator of the unitary transformation $\eta(l)$ is

$$
\eta(l)=\partial_{l} U^{\dagger}(l) U(l)=-U^{\dagger}(l) \partial_{l} U(l) .
$$

Let us remark that the spirit is a bit different to what has been done with the Bogoliubov transform. Indeed here, $H(\infty)$ is unitary equivalent to $H(0)$ and is diagonal in the original basis in which $H(0)$ is nondiagonal. Conversely, when using the Bogoliubov transform, we expressed the same Hamiltonian $H(0)$ in two different basis (the $a$ 's and the $b$ 's).

One must also simultaneously keep track of the change of basis on other operators in which one is interested. Denoting by $\Omega=\Omega(l=0)$ such an operator in the original basis, one defines the flowing operator

$$
\Omega(l)=U^{\dagger}(l) \Omega(0) U(l),
$$

which is subject to the same flow equation as $H$, namely,

$$
\partial_{l} \Omega(l)=[\eta(l), \Omega(l)] .
$$

This allows one to compute the expectation value of any operator $\Omega$ on an eigenstate $|\psi\rangle$ of $H$. Indeed, one has

$$
\langle\psi|\Omega| \psi\rangle=\left\langle\psi\left|U(l=\infty) \Omega(l=\infty) U^{\dagger}(l=\infty)\right| \psi\right\rangle,
$$

where $U^{\dagger}(l=\infty)|\psi\rangle$ is simply the eigenstate of the diagonal Hamiltonian $H(l=\infty)$. 
We still have to give a prescription for the antihermitian generator $\eta(l)$, that has to be chosen to bring the final Hamiltonian into a diagonal form. Wegner proposed $\eta(l)=\left[H_{\mathrm{d}}(l), H_{\mathrm{od}}(l)\right]=\left[H_{\mathrm{d}}(l), H(l)\right]$, where $H_{\mathrm{d}}$ and $H_{\mathrm{od}}$ are the diagonal and off-diagonal part of the Hamiltonian. Other generators have been proposed, and we will use the so-called quasiparticle conserving generator which is better adapted to our problem. We shall now give the motivation for this choice and then derive the flow equations.

\section{B. The flow equations}

When expressed in terms of the bosonic operators $a$ and $a^{\dagger}$, the initial Hamiltonian (28) reads

$$
H(0)=H_{0}(0)+H_{1}^{+}(0)+H_{1}^{-}(0)+H_{2}^{+}(0)+H_{2}^{-}(0),
$$

where $H_{1,2}^{-}=\left(H_{1,2}^{+}\right)^{\dagger}$ and 0,1 , or 2 subscripts indicate the number of created $(+)$ or annihilated $(-)$ excitations. When using Wegner's generator, one would take $H_{0}(l)$ as the diagonal part and the rest as the off-diagonal part so that the generator would be

$$
\eta(l)=\left[H_{0}(l), H_{1}^{+}(l)+H_{1}^{-}(l)+H_{2}^{+}(l)+H_{2}^{-}(l)\right] .
$$

Such a choice of generator suffers from one major drawback: the flowing Hamiltonian $H(l)$ does not remain band diagonal as it is initially, but contains terms creating or annihilating any number of excitations $H_{k}^{ \pm}, k \geq 1$.

A possible choice of generator avoiding this problem is the so-called quasiparticle conserving generator first proposed by Mielke $\frac{45}{5}$ in the context of finite matrices and generalized to many-body physics by Knetter and Uhrig ${ }^{46}$. For the problem at hand, this generator reads

$$
\eta(l)=H_{1}^{+}(l)-H_{1}^{-}(l)+H_{2}^{+}(l)-H_{2}^{-}(l),
$$

and coincides in the symmetric phase $\left(H_{1}^{ \pm}=0\right)$ with the generator proposed by Stein 47 .

From a practical point of view, this generator is also more convenient than Wegner's generator because one does not have to compute a commutator to find the generator. In addition, the flow equations are quadratic instead of cubic in the Hamiltonians

$$
\begin{aligned}
\partial_{l} H_{0}(l) & =2\left(\left[H_{1}^{+}(l), H_{1}^{-}(l)\right]+\left[H_{2}^{+}(l), H_{2}^{-}(l)\right]\right), \\
\partial_{l} H_{1}^{+}(l) & =\left[H_{1}^{+}(l), H_{0}(l)\right]+2\left[H_{2}^{+}(l), H_{1}^{-}(l)\right], \\
\partial_{l} H_{2}^{+}(l) & =\left[H_{2}^{+}(l), H_{0}(l)\right] .
\end{aligned}
$$

One can furthermore check, for example, that no three particle term is generated during the flow since the sum of $\left[H_{1}^{+}(l), H_{2}^{+}(l)\right]$ and $\left[H_{2}^{+}(l), H_{1}^{+}(l)\right]$ judiciously cancels.

Equations (71)- (73) are unexploitable as they stand, because we can only follow the flow of coupling constants, not of operators, and because the $1 / N$ expansion is not

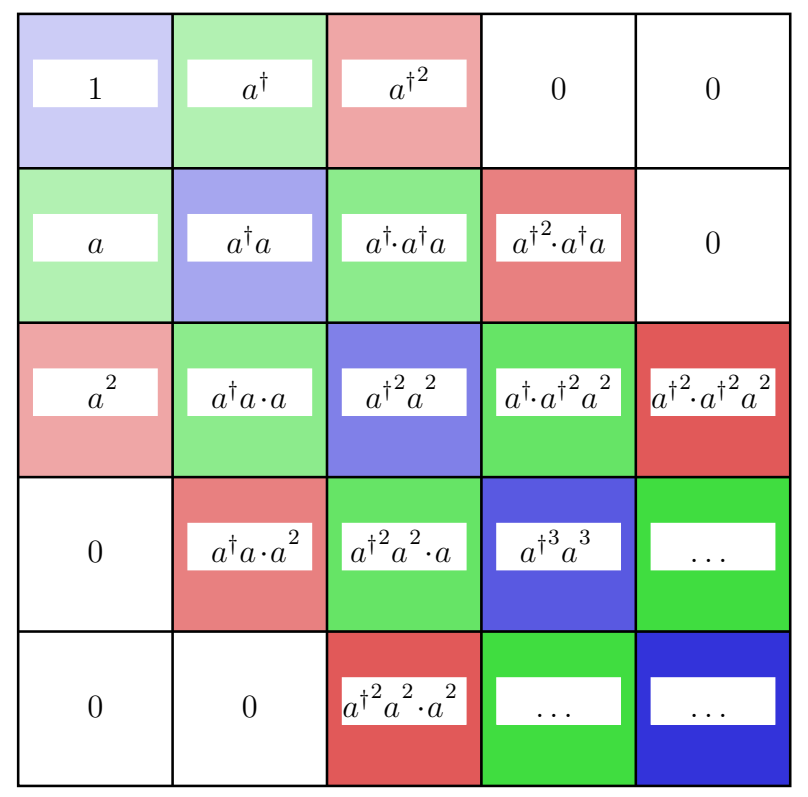

FIG. 5: Structure of the Hamiltonian in the bosonic operators $a$ and $a^{\dagger}$, for terms involving less than six $a$ or $a^{\dagger}$ operators only (see the main text).

yet explicit. Both points are remedied writing

$$
\begin{aligned}
H_{0}(l) & =\sum_{\alpha, \delta \in \mathbb{N}} \frac{h_{0, \alpha}^{(\delta)}(l) A_{\alpha}}{N^{\alpha+\delta-1}}, \\
H_{1}^{+}(l) & =\sum_{\alpha, \delta \in \mathbb{N}} \frac{h_{1, \alpha}^{(\delta)}(l) a^{\dagger} A_{\alpha}}{N^{\alpha+\delta-1 / 2}}, \\
H_{2}^{+}(l) & =\sum_{\alpha, \delta \in \mathbb{N}} \frac{h_{2, \alpha}^{(\delta)}(l) a^{\dagger^{2}} A_{\alpha}}{N^{\alpha+\delta}},
\end{aligned}
$$

where $A_{\alpha}=a^{\dagger^{\alpha}} a^{\alpha}$. The couplings $h_{k, \alpha}^{(\delta)}(l)$ are precisely the flowing quantities we wish to follow. The $k=0,1,2$ subscript is associated to the number of created excitations $a^{\dagger}$. The $\alpha$ subscript keeps track of the number of $a^{\dagger}$ and $a$ operators in the $A_{\alpha}$ operator. Finally, the $\delta$ superscript codes for the successive $1 / N$ corrections. For example, the diagonal part of the Hamiltonian is

$$
\begin{aligned}
H_{0}(l)= & N h_{0,0}^{(0)}(l)+h_{0,0}^{(1)}(l)+\frac{1}{N} h_{0,0}^{(2)}(l)+\ldots \\
& +\left[h_{0,1}^{(0)}(l)+\frac{1}{N} h_{0,1}^{(1)}(l)+\ldots\right] a^{\dagger} a \\
& +\left[\frac{1}{N} h_{0,2}^{(0)}(l)+\ldots\right] a^{\dagger^{2}} a^{2} \\
& +\ldots,
\end{aligned}
$$

where only terms up to order $1 / N$ have been written explicitely.

To make things even more concrete, let us write the 
initial conditions $(l=0)$

$$
\begin{aligned}
& h_{0,0}^{(0)}(0)=\frac{-1-2 h m+m^{2}}{2}=e_{0}(\infty), \\
& h_{0,0}^{(1)}(0)=\frac{1-m^{2}}{2}=\delta e_{0}^{(\mathrm{B})}, \\
& h_{0,0}^{(2)}(0)=0, \\
& h_{0,1}^{(0)}(0)=2+2 h m-3 m^{2}-\gamma=\Delta^{(\mathrm{B})}(\infty), \\
& h_{0,1}^{(1)}(0)=-2+3 m^{2}+\gamma, \\
& h_{0,2}^{(0)}(0)=-2+3 m^{2}+\gamma,
\end{aligned}
$$

and all coefficients of higher order vanish.

We have depicted the structure of the Hamiltonian in Fig. 5 for all terms involving less than six $a$ or $a^{\dagger}$ operators. Let us emphasize that this figure does not represent the Hamiltonian in the eigenbasis $\{|n\rangle\}$ of the number operator $a^{\dagger} a$. Let us explain on the example of the operator $a^{\dagger} \cdot a^{\dagger^{2}} a^{2}$ how it should be interpreted. This operator annihilates the states $|0\rangle$ and $|1\rangle$ containing zero or one excitation. The first state it does not annihilate is $|2\rangle$ and its action is to increase the number of excitations by one, and so gives a state proportional to $|3\rangle$. For these reasons, this operator is written on the first upper offdiagonal, at the position $(3,2)$. It is, however, clear that this many-body operator also acts nontrivially on any state $|n \geq 2\rangle$. As another example, the zeroes shown in the figure mean that the Hamiltonian does not contain terms creating three or more excitations.

Equations (74)- (76) can be inserted in (7173) to give the flow equations of the various $h_{k, \alpha}^{(\delta)}(l)$ couplings

$$
\begin{aligned}
\partial_{l} h_{0, \alpha}^{(\delta)}(l) & =2 \sum_{n, \alpha^{\prime}, \delta^{\prime}} \mathcal{C}_{\alpha^{\prime}, \alpha-\alpha^{\prime}-1+n}^{1,1, n} h_{1, \alpha^{\prime}}^{\left(\delta^{\prime}\right)}(l) h_{1, \alpha-\alpha^{\prime}-1+n}^{\left(\delta-\delta^{\prime}+1-n\right)}(l)+\sum_{n, \alpha^{\prime}, \delta^{\prime}} \mathcal{C}_{\alpha^{\prime}, \alpha-\alpha^{\prime}-2+n}^{2,2, n} h_{2, \alpha^{\prime}}^{\left(\delta^{\prime}\right)}(l) h_{2, \alpha-\alpha^{\prime}-2+n}^{\left(\delta-\delta^{\prime}+1-n\right)}(l), \\
\partial_{l} h_{1, \alpha}^{(\delta)}(l) & =\sum_{n, \alpha^{\prime}, \delta^{\prime}} \mathcal{C}_{\alpha^{\prime}, \alpha-\alpha^{\prime}+n}^{1,0, n} h_{1, \alpha^{\prime}}^{\left(\delta^{\prime}\right)}(l) h_{0, \alpha-\alpha^{\prime}+n}^{\left(\delta-\delta^{\prime}+1-n\right)}(l)+2 \sum_{n, \alpha^{\prime}, \delta^{\prime}} \mathcal{C}_{\alpha^{\prime}, \alpha-\alpha^{\prime}-1+n}^{2,1, n} h_{2, \alpha^{\prime}}^{\left(\delta^{\prime}\right)}(l) h_{1, \alpha-\alpha^{\prime}-1+n}^{\left(\delta-\delta^{\prime}+1-n\right)}(l) \\
\partial_{l} h_{2, \alpha}^{(\delta)}(l) & =\sum_{n, \alpha^{\prime}, \delta^{\prime}} \mathcal{C}_{\alpha^{\prime}, \alpha-\alpha^{\prime}+n}^{2,0, n} h_{2, \alpha^{\prime}}^{\left(\delta^{\prime}\right)}(l) h_{0, \alpha-\alpha^{\prime}+n}^{\left(\delta-\delta^{\prime}+1-n\right)}(l) .
\end{aligned}
$$

The $\mathcal{C}$ coefficients arise from the computation of the commutators

$$
\begin{aligned}
& {\left[a^{\dagger j^{\prime}} A_{\alpha^{\prime}}, A_{\alpha^{\prime \prime}} a^{j^{\prime \prime}}\right]}
\end{aligned}
$$

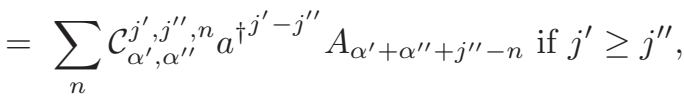

$$
\begin{aligned}
& =\sum_{n} \mathcal{C}_{\alpha^{\prime}, \alpha^{\prime \prime}}^{j^{\prime} j^{\prime \prime}, n} A_{\alpha^{\prime}+\alpha^{\prime \prime}+j^{\prime}-n} a^{j^{\prime \prime}-j^{\prime}} \text { if } j^{\prime} \leq j^{\prime \prime},
\end{aligned}
$$

and are equal to

$$
\mathcal{C}_{\alpha^{\prime}, \alpha^{\prime \prime}}^{j^{\prime}, j^{\prime \prime}, n}=n !\left(C_{\alpha^{\prime}}^{n} C_{\alpha^{\prime \prime}}^{n}-C_{\alpha^{\prime}+j^{\prime}}^{n} C_{\alpha^{\prime \prime}+j^{\prime \prime}}^{n}\right),
$$

$C_{\alpha}^{n}$ being the usual binomial coefficient $\alpha ! /[n !(\alpha-n) !]$. The various sums in (84) - 86 are constrained by the fact that all subscripts and superscripts have to be positive. Thus for example, in the first sum of Eq. (84), $n$ runs from 0 to $1+\delta, \alpha^{\prime}$ from 0 to $\alpha-1+n$, and $\delta^{\prime}$ from 0 to $\delta+1-n$.

We shall now explain how to diagonalize the Hamiltonian order by order in $1 / N$. The most important point is to ensure that corrections to a given order are unchanged when going to next orders. This means that terms of order $(1 / N)^{-1}$ and $(1 / N)^{-1 / 2}$ must not appear in the flow equations. Here, this is indeed the case because (i) the term of order $(1 / N)^{-1}$ is proportional to the identity operator and (ii) the term of order $(1 / N)^{-1 / 2}$ has been suppressed by the appropriate choice of $\theta_{0}$. Consequently, couplings of order $(1 / N)^{-1}$ and $(1 / N)^{-1 / 2}$ are simply $h_{0,0}^{(0)}(l)=e_{0}(\infty)$ and $h_{1,0}^{(0)}(l)=0$ for all $l$.

At the next order $(1 / N)^{0}$, Eqs. [84)-86 give

$$
\begin{aligned}
& \partial_{l} h_{0,0}^{(1)}(l)=-4 h_{2,0}^{(0)^{2}}(l) \\
& \partial_{l} h_{0,1}^{(0)}(l)=-8{h_{2,0}^{(0)^{2}}(l)}^{(0)}=-2 h_{0,1}^{(0)}(l) h_{2,0}^{(0)}(l) . \\
& \partial_{l} h_{2,0}^{(0)}(l)=
\end{aligned}
$$

These equations are simply the Bogoliubov transformation, written in a differential form ${ }^{44}$. It is a simple matter to recover Eqs. (41) and (42) from the two constants of the flow, $2 h_{0,0}^{(1)}(l)-h_{0,1}^{(0)}(l)$ and $h_{0,1}^{(0)}(l)^{2}-4 h_{2,0}^{(0)}(l)^{2}$.

The same type of calculation can be performed for the observables $\tilde{S}_{x}, \tilde{S}_{y}$ and $\tilde{S}_{z}$ as well as the correlation functions $\tilde{S}_{\alpha} \tilde{S}_{\beta}$. As the analysis closely resembles the one we performed for the Hamiltonian, and as we want the main text to be as fluid as possible, we have gathered the flow equations of these observables and their derivations in Appendix A Let us simply mention that contrarily to the Hamiltonian, the observables do not retain the simple structure they exhibit in the beginning of the flow, even when using the quasiparticle conserving generator.

The flow equations for the Hamiltonian and the observables can be integrated exactly in both phases, at 
least up to the orders considered in this work. From the solutions, one can compute the renormalized quantities (i.e., the values at $l=\infty$ ). Note that the possibility to get exact $1 / N$ corrections may originate from the integrability of the LMG model and is not a generic feature of the CUTs. We explain how to compute the solutions to the flows of the energies and observables in Appendix B The results for the renormalized quantities are gathered

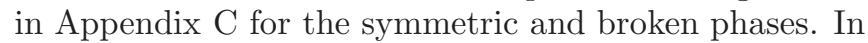
the next subsection we explain how to make use of these results to compute finite-size scaling exponents.

\section{Computation of finite-size scaling exponents}

In this section, we explain how to make use of the results of the $1 / N$ expansion to compute the finite-size exponents of the spectrum and of the correlation functions of the anisotropic model. Let us first focus on the results obtained in the symmetric phase, see Appendix C2 ¿From Eqs. (C3), (C10, (C17), C23), C28), C33) one sees that any physical quantities $\Phi$ that we have computed can be written as

$$
\Phi_{N}(h, \gamma)=\Phi_{N}^{\mathrm{reg}}(h, \gamma)+\Phi_{N}^{\mathrm{sing}}(h, \gamma)
$$

where the superscripts "reg" and "sing" stands for regular and singular, respectively. A nonsingular contribution is understood to be a function of $h$ which is nonsingular at $h=1$, as well as all its derivatives. As an example, if $\Phi=2\left\langle S_{z}\right\rangle / N$ [see Eq. [C17]], the regular part is $1+1 / N$ and the remaining forms the singular part. Now let us suppose $h$ is close to its critical value 1 . Then all terms involving the polynomial functions $Q_{\Phi}^{(i)}$ become small compared to the terms involving the $P_{\Phi}^{(i)}$ s, by a factor $\Xi(h, \gamma)=(h-1)(h-\gamma)$. In this limit, one can write

$$
\Phi_{N}^{\mathrm{sing}}(h, \gamma) \simeq \frac{\Xi(h, \gamma)^{\xi_{\Phi}}}{N^{n_{\Phi}}} \mathcal{F}_{\Phi}\left[N \Xi(h, \gamma)^{3 / 2}, \gamma\right]
$$

where $\mathcal{F}_{\Phi}$ is the scaling function for the physical quantity $\Phi$, depending only on the scaling variable $N \Xi(h, \gamma)^{3 / 2}$ [but not separately on $N$ and $\Xi(h, \gamma)$ ], $\xi_{\Phi}$ and $n_{\Phi}$ are exponents that we list in Table \ Now we use the fact

\begin{tabular}{||c|c|c|c||}
\hline \hline$\Phi$ & $\xi_{\Phi}$ & $n_{\Phi}$ & $n_{\Phi}+2 \xi_{\Phi} / 3$ \\
\hline \hline$e_{0}$ & $1 / 2$ & 1 & $4 / 3$ \\
\hline$\Delta$ & $1 / 2$ & 0 & $1 / 3$ \\
\hline $2\left\langle S_{z}\right\rangle / N$ & $-1 / 2$ & 1 & $2 / 3$ \\
\hline $4\left\langle S_{x}^{2}\right\rangle / N^{2}$ & $-1 / 2$ & 1 & $2 / 3$ \\
\hline $4\left\langle S_{y}^{2}\right\rangle / N^{2}$ & $1 / 2$ & 1 & $4 / 3$ \\
\hline $4\left\langle S_{z}^{2}\right\rangle / N^{2}$ & $-1 / 2$ & 1 & $2 / 3$ \\
\hline \hline
\end{tabular}

TABLE I: Table of exponents (see the text for their signification) for the physical quantities $\Phi$ considered in this work. that there can be no singularity in any physical quantity in a finite-size system. This implies that the singularity of $\Xi(h, \gamma)^{\xi_{\Phi}}$ has to be canceled by the one of $\mathcal{F}_{\Phi}\left[N \Xi(h, \gamma)^{3 / 2}, \gamma\right]$. Thus one must have $\mathcal{F}_{\Phi}[x, \gamma] \sim$ $x^{-2 \xi_{\Phi} / 3}$, which in turn implies the following finite-size scaling

$$
\Phi_{N}^{\text {sing }}(h=1, \gamma) \sim \frac{a_{\Phi}}{N^{n_{\Phi}+2 \xi_{\Phi} / 3}},
$$

where the exponents $n_{\Phi}+2 \xi_{\Phi} / 3$ are listed in the last column of Table【 and the $a_{\Phi}$ 's are constants which cannot be determined with this scaling argument. These finitesize exponents are compatible with the values obtained from numerical diagonalization and discussed in the next section (see Figs. [6] and 7).

Let us finally mention that the exponents for the ground-state energy per spin $e_{0}$ and for the gap $\Delta$ can also be computed following the same line of reasoning, from the results obtained in the broken phase [see Eqs. [C39) and (C40)]. The only difference is that one has to replace the variable $\Xi(h, \gamma)=(h-1)(h-\gamma)$ by $\Psi(h)=1-h^{2}$. For the observables we did not go to sufficiently high order to perform the same analysis. However, we can argue that from the results for $e_{0}$ and $\Delta$ that the scaling variable is $N \Psi(h)^{3 / 2}$. Then, one can easily show that we recover all exponents listed in Table \

\section{NUMERICAL RESULTS}

In order to check the validity of the approach used here to compute the critical exponents, we have numerically diagonalized $H$ in the sector $S=N / 2$ where the lowenergy states lie. The dimension of this subspace is $N+1$ but the spin-flip symmetry (4) further divides by 2 the dimension of the matrices to be diagonalized. These simplifications allows one to easily investigate rather large system size (up to $N=2^{20}$ spins for the energy spectrum and $N=2^{14}$ spins for observables requiring eigenstates). For each system size $N$, we have computed the groundstate energy $e_{0}$, the energy gap $\Delta$, the magnetization $\left\langle S_{z}\right\rangle$ and the correlation functions $\left\langle S_{x}^{2}\right\rangle,\left\langle S_{y}^{2}\right\rangle$, and $\left\langle S_{z}^{2}\right\rangle$ at the critical point $h=1$. For clarity, we only present the results for $\gamma=0$ but we have checked that the scaling exponents are the same for any $\gamma \neq 1$ as expected from our analysis, the peculiar case $\gamma=1$ being discussed in Sec. IIB

As can be seen in Fig. [6 the leading nontrivial finitesize corrections for $e_{0}$ and $\Delta$ are indeed proportional to $N^{-4 / 3}$ and $N^{-1 / 3}$. Note that this latter result was already observed numerically by Botet and Jullien ${ }^{30}$ twenty years ago but, to our knowledge, never proven by analytical arguments.

Our results are in contradiction with a recent study by Reslen and co-workers $\frac{15}{5}$ who computed numerically some correlation functions. They indeed obtained that $2\left\langle S_{z}^{2}\right\rangle / N-1 \sim N^{-0.55 \pm 0.01}$. The source of this discrepancy comes from the too small system size they investi- 


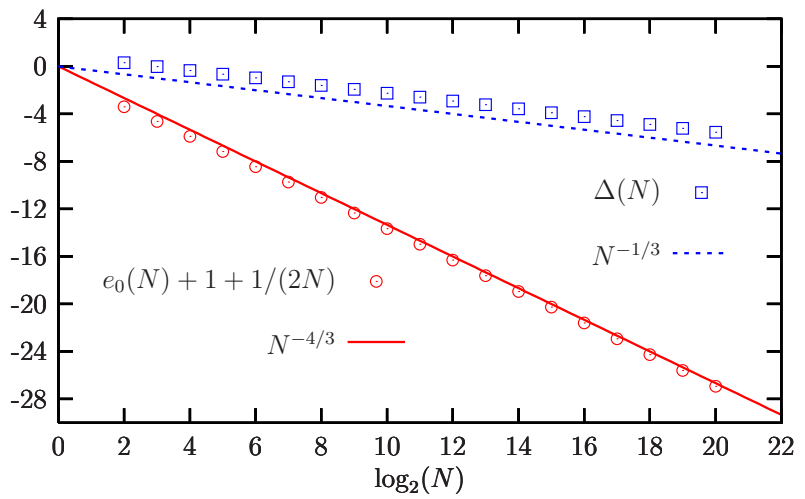

FIG. 6: $\log _{2}-\log _{2}$ plot of the ground-state energy per spin $e_{0}$ and the enery gap $\Delta$ at the critical point $h=1$ as a function of $N$, for $\gamma=0$.

gated $(100 \leq N \leq 500)$. Indeed, in this range, we have checked that the scaling exponent is indeed close to 0.55 , but for larger size as those considered here $\left(N \sim 2^{14}\right)$, we found numerically that the fitted exponent gets closer and closer from $2 / 3$ which is the value predicted by our approach.

\section{ENTANGLEMENT PROPERTIES}

The entanglement properties in the LMG model have recently attracted much attention $15,21,31,32,33$. For the ferromagnetic case considered here, several quantities have been used to characterize the ground-state entanglement. First, the concurrence $\frac{48}{2}$ which measures the two-spin entanglement has been shown to develop a cusp at the critical point ${ }^{31}$ with a nontrivial scaling behavior that we shall discuss below. Secondly, the von Neumann entropy has also been computed as a function of both the anisotropy and the magnetic field 21 . Its scaling at the critical point has suggested the possibility of an underlying conformal theory describing the LMG model with central charges $c=3 / 2$ for $\gamma=1$ and $c=1$ otherwise. Finally, we wish to clarify the situation about another measure proposed by Somma et al $\stackrel{49}{\underline{4}}$, the so-called $\mathrm{SU}(2)$-purity. For a system where all spins are equivalent as in the LMG model, this quantity is nothing but the square of the reduced Bloch sphere radius defined by

$$
r^{2}=\left\langle\sigma_{x}\right\rangle^{2}+\left\langle\sigma_{y}\right\rangle^{2}+\left\langle\sigma_{z}\right\rangle^{2}=\left(\frac{2\left\langle S_{z}\right\rangle}{N}\right)^{2}
$$

The latter relation is due to the spin-flip symmetry (4) and is only valid for the exact finite- $N$ ground state. It is thus simply connected to the magnetization in the field direction so that the SU(2)-purity does not bring any new information about the entanglement of the ground state in this model. Furthermore, this purity is trivially computed in the thermodynamical limit using the semiclassical description discussed in Sec. IIII Unfortunately, for the

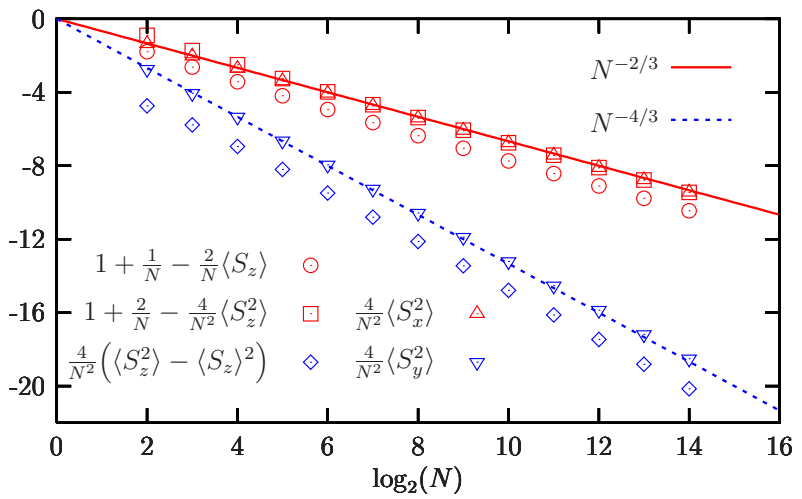

FIG. 7: Scaling of various observables as a function of $N$ $\left(\log _{2}-\log _{2}\right.$ plot) at the critical point $h=1$, for $\gamma=0$..

microscopic degrees of freedom which are the spins half, there is no entanglement in this limit since the ground state is a pure product state [see ansatz (19)]. Clearly, Somma et al. did not understand that the richness of the entanglement in this model was given by the finite- $N$ corrections which we shall now discuss in the framework of the concurrence.

Introduced by Wootters 48 , the concurrence which measures the entanglement between two spins half is defined as follows. For a given pure state $|\psi\rangle$, we define the density matrix $\rho=|\psi\rangle\langle\psi|$ and the two-spin reduced density matrix $\rho_{i, j}$ obtained by tracing out $\rho$ over all spins except spins $i$ and $j$. Next, we introduce the spin-flipped density matrix $\tilde{\rho}_{i, j}=\left(\sigma_{y} \otimes \sigma_{y}\right) \rho_{i, j}^{*}\left(\sigma_{y} \otimes \sigma_{y}\right)$ where $\rho_{i, j}^{*}$ is the complex conjugate of $\rho_{i, j}$. The concurrence is then defined by

$$
C_{i, j}=\max \left\{0, \mu_{1}-\mu_{2}-\mu_{3}-\mu_{4}\right\},
$$

where the $\mu_{k}$ 's are the square roots of the four real eigenvalues of $\rho_{i, j} \tilde{\rho}_{i, j}$, and where $\mu_{i} \geq \mu_{i+1}$. The concurrence vanishes if the reduced density matrix $\rho_{i, j}$ can be decomposed into unentangled pure states whereas it reaches its maximal values 1 for maximally entangled (two-spin) states such as the famous Einstein-Podolsky-Rosen state

$$
|\mathrm{EPR}\rangle=\frac{1}{\sqrt{2}}(|\uparrow \downarrow\rangle-|\downarrow \uparrow\rangle) .
$$

In the LMG model, since all spins are equivalent, the concurrence does not explicitely depend on $i$ and $j$ and we shall omit these indexes in the following. In addition, since the ground state lies in the sector $S=N / 2$ the concurrence can be easily expressed in terms of the observables computed in the previous section. Indeed, for any symmetric state, the reduced density matrix reads 50 :

$$
\rho=\left(\begin{array}{llll}
v_{+} & x_{+}^{*} & x_{+}^{*} & u^{*} \\
x_{+} & y & y & x_{-}^{*} \\
x_{+} & y & y & x_{-}^{*} \\
u & x_{-} & x_{-} & v_{-}
\end{array}\right)
$$


with

$$
\begin{aligned}
v_{ \pm} & =\frac{N^{2}-2 N+4\left\langle S_{z}^{2}\right\rangle \pm 4(N-1)\left\langle S_{z}\right\rangle}{4 N(N-1)} \\
x_{ \pm} & =\frac{(N-1)\left\langle S_{+}\right\rangle \pm\left\langle\left[S_{+}, S_{z}\right]_{+}\right\rangle}{2 N(N-1)} \\
y & =\frac{N^{2}-4\left\langle S_{z}^{2}\right\rangle}{4 N(N-1)} \\
u & =\frac{\left\langle S_{+}^{2}\right\rangle}{N(N-1)} .
\end{aligned}
$$

For an eigenstate of the spin-flip operator as, for instance, the ground state of the LMG model at finite $N$, one further has $x_{ \pm}=0$, so that the concurrence is simply given by ${ }^{50}$

$$
C=\left\{\begin{array}{l}
2 \max (0,|u|-y) \text { if } 2 y \leq \sqrt{v_{+} v_{-}}+|u| \\
2 \max \left(0, y-\sqrt{v_{+} v_{-}}\right) \text {if } 2 y \geq \sqrt{v_{+} v_{-}}+|u| .
\end{array}\right.
$$

\section{A. The thermodynamical limit}

Using the expressions of the observables gathered in Secs. C1 and C2 one can show that for $\sqrt{\gamma} \leq h$, one has $2 y \leq \sqrt{v_{+} v_{-}}+|u|$ so that the rescaled concurrence $C_{\mathrm{R}}=(N-1) C$ reads $^{34}$

$$
C_{\mathrm{R}}^{\sqrt{\gamma} \leq h}=\frac{2}{N}\left(\left|\left\langle S_{x}^{2}-S_{y}^{2}\right\rangle\right|-N^{2} / 4+\left\langle S_{z}^{2}\right\rangle\right),
$$

which can be rewritten as

$$
C_{\mathrm{R}}^{\sqrt{\gamma} \leq h}=1-\frac{4\left\langle S_{y}^{2}\right\rangle}{N}
$$

since for $|\gamma| \leq 1$, one has $\left|\left\langle S_{x}^{2}-S_{y}^{2}\right\rangle\right| \geq 0$. It is worth noting that, obviously, the concurrence $C$ goes to zero in the thermodynamic limit since the ground state becomes a product state. However, the relevant (nontrivial) quantity for this problem is the rescaled concurrence $C_{\mathrm{R}}$. This rescaling takes into account the fact that the entanglement is equally shared between all spins and shall be interpreted as the connectivity of each spin. Using expression (61) obtained by the Bogoliubov transform, one can then compute the asymptotic behavior of the concurrence in both phases. One thus has

$$
\begin{aligned}
C_{\mathrm{R}}^{h \geq 1} & =1-\sqrt{\frac{h-1}{h-\gamma}}, \\
C_{\mathrm{R}}^{\sqrt{\gamma} \leq h \leq 1} & =1-\sqrt{\frac{1-h^{2}}{1-\gamma}} .
\end{aligned}
$$

These expressions are valid for any $|\gamma|<1$ and coincides, for $\gamma=0$, with the results found by Reslen et al. 15 . They also prove that a real cusplike singularity occurs at the critical point as inferred from numerical results in Ref 31 . Note that in the broken phase, the concurrence vanishes for $h=\sqrt{\gamma}$ which is precisely the point where the ground state is a pure product state at finite $N$ (see Sec. III). At this special point one has $2 y=\sqrt{v_{+} v_{-}}+|u|$ and for $h \leq \sqrt{\gamma}$, one finds that $2 y \geq \sqrt{v_{+} v_{-}}+|u|$ so the second expression in Eq. 103 must be considered to compute the concurrence, namely

$$
C_{\mathrm{R}}^{h \leq \sqrt{\gamma}}=\frac{1}{2 N}\left\{N^{2}-4\left\langle S_{z}^{2}\right\rangle-\sqrt{\left[N(N-2)+4\left\langle S_{z}^{2}\right\rangle\right]^{2}-\left[4(N-1)\left\langle S_{z}\right\rangle\right]^{2}}\right\}
$$

Using the expansion of $\left\langle S_{z}\right\rangle$ (C1C and $\left\langle S_{z}^{2}\right\rangle$ (C1fl), one then finds:

$$
C_{\mathrm{R}}^{h \leq \sqrt{\gamma}}=1-\sqrt{\frac{1-\gamma}{1-h^{2}}}
$$

We emphasize that for $h=0$, this latter expression shows that the rescaled concurrence of the ground state is already nontrivial since it only vanishes for $\gamma=0$. Furthermore, let us stress that this result is beyond the reach of the Bogoliubov transform. The results for the rescaled concurrence in the thermodynamical limit are summarized in Fig. 8 for an anisotropy parameter $\gamma=1 / 2$.

It is important to note that, in the broken phase, we have used the expressions of the correlation functions computed in a $1 / N$ expansion around the ansatz state $\left|\psi\left(\theta_{0}, \phi_{0}\right)\right\rangle$ [see Eq. (19)] which is not the exact ground state since it has, in particular, nonvanishing values of $\left\langle S_{x}\right\rangle$ and $\left\langle S_{y}\right\rangle$. The exact ground state $\left|\psi_{0}\right\rangle$ and the exact first excited state $\left|\psi_{1}\right\rangle$ indeed belong to subspaces of fixed and different spin-flip symmetry. However, in the broken phase, correlation functions for these states are identical up to exponentially small terms $\sim \exp (-a N)$. Thus, for any state $\left|\Psi_{\alpha}\right\rangle=\cos \alpha\left|\psi_{0}\right\rangle+\sin \alpha\left|\psi_{1}\right\rangle$, the average of an operator $\Omega$ such that $\left[\Omega, \prod_{i} \sigma_{z}^{i}\right]=0$ (as, for instance, $S_{y}^{2}, S_{z}$ or $S_{z}^{2}$ ) is independent of $\alpha$ and satisfies

$$
\langle\Psi(\alpha)|\Omega| \Psi(\alpha)\rangle=\left\langle\psi_{0}|\Omega| \psi_{0}\right\rangle=\left\langle\psi_{1}|\Omega| \psi_{1}\right\rangle .
$$




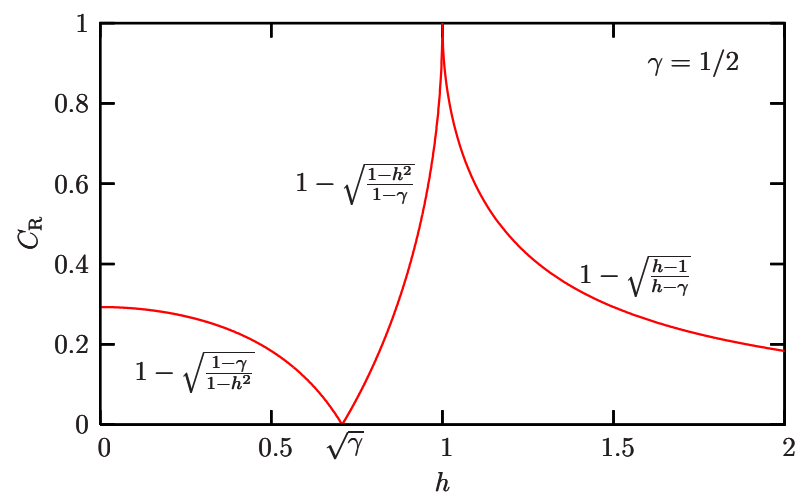

FIG. 8: Rescaled concurrence as a function of the magnetic field, in the thermodynamical limit, and for $\gamma=1 / 2$.

This identity is valid up to exponentially small terms and justifies our use of correlation functions computed from the $1 / N$ expansion around one of the two broken symmetry state.

\section{B. Finite- $N$ corrections}

Let us now discuss the finite- $N$ corrections to the rescaled concurrence. For $|\gamma|<1$, apart from the critical point, the leading corrections are proportionnal to $1 / N$ since the expansion of observables is not singular. A contrario, at $h=1$, using Eq. 105) and results of Sec. C1e one gets

$$
C_{\mathrm{R}}^{h=1} \sim 1-a_{y y} N^{-1 / 3} .
$$

which is indeed the result suggested in Refs ${ }^{15.31}$. We have summarized these scalings in Fig. 9 for $\gamma=0$.

\section{The isotropic case}

For completeness, let us briefly discuss the isotropic case. As explained in Sec. IIB for $\gamma=1$, the ground state is simply the Dicke state $\left.\left\{N / 2, M_{0}\right\rangle\right\}$ whose rescaled concurrence is

$$
\begin{aligned}
C_{\mathrm{R}}= & \frac{1}{2 N}\left\{N^{2}-4 M_{0}^{2}-\right. \\
& \left.\sqrt{\left(N^{2}-4 M_{0}^{2}\right)\left[(N-2)^{2}-4 M_{0}^{2}\right]}\right\} .
\end{aligned}
$$

In the thermodynamical limit, one thus has a discontinuity at the critical point where the rescaled concurrence jumps from 0 (for $h>1$ ) to 2 for $(h<1)$, and goes to 1 in the zero field limit. Let us finally mention that this behavior can not be recovered by taking the limit $\gamma \rightarrow 1$ of the anisotropic results. Indeed, although Eq. (106) properly predicts $C_{\mathrm{R}}=0$ in the symmetric phase, the broken phase result (107) diverges in the limit $\gamma \rightarrow 1$. The physical origin of this divergence is the presence of a

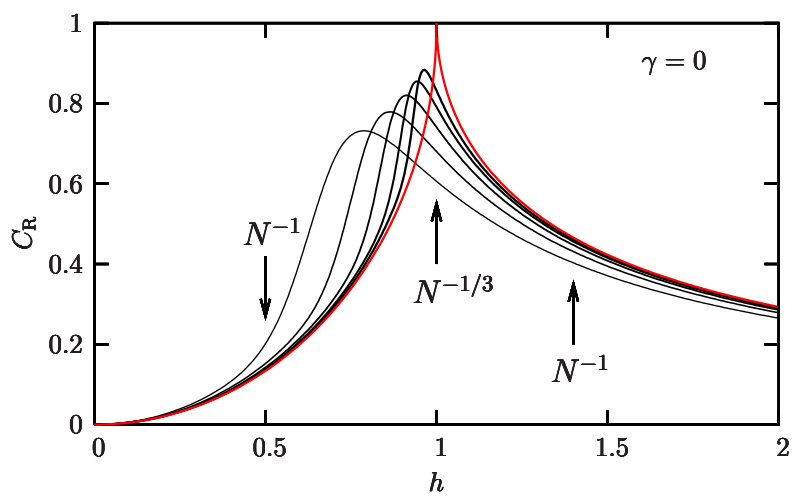

FIG. 9: Rescaled concurrence as a function of the magnetic field, for $\gamma=0$, and for system sizes $N=16,32,64,128,256$ and $\infty$ (bottom to top). Arrows indicate the behavior of the finite size correction in various regions.

Goldstone mode associate to the broken rotational symmetry along the $z$ axis.

\section{CONCLUSION}

The aim of this work was mainly twofold. We have computed the finite-size scaling exponents for the spectrum as well as one- and two-spin correlation functions of the quantum critical LMG model. We have also characterized the two-spin entanglement properties of its ground state by computing the concurrence.

Such studies had already been performed numerically, by computing the exact eigenenergies and eigenvectors. The numerical calculations are not too demanding since the size of the matrix to be diagonalized grows linearly with the number of spins $N$. Such numerical results were presented in Sec. V1] where we computed the eigenenergies for system sizes as large as $N=2^{20}$, and the correlation functions for less than $N=2^{14}$ spins.

Our main goal however was to provide an analytical derivation of the simple finite-size scaling exponents observed numerically (Sec. VC), as well as formulas for the rescaled concurrence in the thermodynamical limit (Sec. VIIA). Both of these calculations require the knowledge of $1 / N$ corrections to the thermodynamical limit (mean-field) solution (Sec. IIII). On the one hand, as far as entanglement properties are concerned, it is clear that one has to go beyond the mean-field wave function, which is separable and can thus display no entanglement. The nontrivial entanglement properties arise in the finitesize system, and the physically interesting property is the first $1 / N$ correction to the concurrence. On the other hand, finite-size scaling exponents at criticality can be extracted from the knowledge of the singularities arising in the $1 / N$ expansion, by making use of a finite-size scaling argument.

In Sec. IV] it was shown that the Bogoliubov transform solution of the bosonic Holstein-Primakoff represen- 
tation of the Hamiltonian truncated to quadratic order does not capture enough of the $1 / N$ corrections needed in this program. One thus has to work with a nonquadratic Hamiltonian which, in turns, means that one needs a method that can solve such interacting Hamiltonians. We have used what we think is the most efficient and elegant method to perform this task, namely the CUTs (Sec. $\nabla \mathrm{A}$ ). With this method, the spectrum and correlation functions are the infinite time limit of some renormalization flows. For the LMG model, such flows can be computed exactly, yielding exact successive $1 / N$ corrections. The integrability of the flows, which is not a generic feature of the CUTs, may originate in the fact that the model is solvable by algebraic Bethe ansatz. One should however stress that it is a difficult task to compute $1 / N$ corrections for the spectrum from the Bethe ansatz solution ${ }^{35.36}$, and even more complicated to do it for correlation functions.

The combination of the $1 / N$ expansion in the HolsteinPrimakoff representation, the CUTs and a scaling argument, opens the possibility to compute finite-size scaling exponents as well as some nontrivial $1 / N$ effects for other models as well. Of particular interest is the reduced BCS model used to describe superconductivity in ultrasmall grains 51 This will be the object of a forthcoming publication. As they stand our results are also directly applicable to the Dicke model, which in the zero-temperature limit can be put in a one-to-one correspondence with the LMG model ${ }^{15}$ We hope more models will turn out to be analyzable by following the same lines of reasoning.

\section{Acknowledgments}

We are indebted to B. Douçot, J. Dukelsky, S. Kirschner, J.-M. Maillard, D. Mouhanna, E. MüllerHartmann, A. Reischl, A. Rosch and K. P. Schmidt for fruitful and valuable discussions. Financial support of the DFG in SP1073 is gratefully acknowledged.

\section{APPENDIX A: FLOW EQUATIONS OF THE OBSERVABLES}

Let us denote by $\Omega(l)$ a general flowing hermitian observable. This observable can be decomposed in the following form:

$$
\Omega(l)=\Omega_{0}(l)+\sum_{k}\left[\Omega_{k}^{+}(l)+\Omega_{k}^{-}(l)\right],
$$

where the sum runs over all nonnegative integers $k$ 's and $\Omega_{k}^{-}=\Omega_{k}^{+\dagger}$. The flow equations are found by computing $[\eta(l), \Omega(l)]$. One finds

$$
\begin{aligned}
\partial_{l} \Omega_{0}(l)= & \left(\left[H_{1}^{+}(l), \Omega_{1}^{-}(l)\right]+\text { h.c. }\right) \quad(\mathrm{A} 2) \\
& +\left(\left[H_{2}^{+}(l), \Omega_{2}^{-}(l)\right]+\text { h.c. }\right), \\
\partial_{l} \Omega_{1}(l)= & {\left[H_{1}^{+}(l), \Omega_{0}(l)\right]+\left[H_{1}^{+}(l), \Omega_{2}^{-}(l)\right]^{\dagger} \quad(\mathrm{A} 3) } \\
& +\left[H_{2}^{+}(l), \Omega_{1}^{-}(l)\right]+\left[H_{2}^{+}(l), \Omega_{3}^{-}(l)\right]^{\dagger}, \\
\partial_{l} \Omega_{2}(l)= & {\left[H_{1}^{+}(l), \Omega_{1}^{+}(l)\right]+\left[H_{1}^{+}(l), \Omega_{3}^{-}(l)\right]^{\dagger} \quad(\mathrm{A} 4) } \\
& +\left[H_{2}^{+}(l), \Omega_{0}(l)\right]+\left[H_{2}^{+}(l), \Omega_{4}^{-}(l)\right]^{\dagger}, \\
\partial_{l} \Omega_{k \geq 3}(l)= & {\left[H_{1}^{+}(l), \Omega_{k-1}^{+}(l)\right]+\left[H_{1}^{+}(l), \Omega_{k+1}^{-}(l)\right]^{\dagger}(\mathrm{A} 5) } \\
& +\left[H_{2}^{+}(l), \Omega_{k-2}^{+}(l)\right]+\left[H_{2}^{+}(l), \Omega_{k+2}^{-}(l)\right]^{\dagger} .
\end{aligned}
$$

In a notation similar to Eqs. (74)-(76), we have introduced

$$
\begin{aligned}
\Omega_{0}(l) & =\sum_{\alpha, \delta \in \mathbb{N}} \frac{\omega_{0, \alpha}^{(\delta)}(l) A_{\alpha}}{N^{\alpha+\delta-1}}, \\
\Omega_{k}^{+}(l) & =\sum_{\alpha, \delta \in \mathbb{N}} \frac{\omega_{k, \alpha}^{(\delta)}(l) a^{\dagger k} A_{\alpha}}{N^{\alpha+\delta+k / 2-1}} .
\end{aligned}
$$

Inserting these formulas into Eqs. A2 - A5 yields

$$
\begin{aligned}
& \partial_{l} \omega_{0, \alpha}^{(\delta)}(l)=2 \sum_{n, \alpha^{\prime}, \delta^{\prime}} \mathcal{C}_{\alpha^{\prime}, \alpha-\alpha^{\prime}-1+n}^{1,1, n} h_{1, \alpha^{\prime}}^{\left(\delta^{\prime}\right)}(l) \Re \omega_{1, \alpha-\alpha^{\prime}-1+n}^{\left(\delta-\delta^{\prime}+1-n\right)}(l)+2 \sum_{n, \alpha^{\prime}, \delta^{\prime}} \mathcal{C}_{\alpha^{\prime}, \alpha-\alpha^{\prime}-2+n}^{2,2, n} h_{2, \alpha^{\prime}}^{\left(\delta^{\prime}\right)}(l) \Re \omega_{2, \alpha-\alpha^{\prime}-2+n}^{\left(\delta-\delta^{\prime}+1-n\right)}(l), \\
& \partial_{l} \omega_{1, \alpha}^{(\delta)}(l)=\sum_{n, \alpha^{\prime}, \delta^{\prime}} \mathcal{D}_{\alpha^{\prime}, \alpha-\alpha^{\prime}+n}^{1,0, n}(l) h_{1, \alpha^{\prime}}^{\left(\delta^{\prime}\right)}(l) \omega_{0, \alpha-\alpha^{\prime}+n}^{\left(\delta-\delta^{\prime}+1-n\right)}(l)+\sum_{n, \alpha^{\prime}, \delta^{\prime}} \mathcal{C}_{\alpha^{\prime}, \alpha-\alpha^{\prime}-1+n}^{1,2, n} h_{1, \alpha^{\prime}}^{\left(\delta^{\prime}\right)}(l) \omega_{2, \alpha-\alpha^{\prime}-1+n}^{\left(\delta-\delta^{\prime}+1-n\right)}{ }^{*}(l) \\
& +\sum_{n, \alpha^{\prime}, \delta^{\prime}} \mathcal{C}_{\alpha^{\prime}, \alpha-\alpha^{\prime}-1+n}^{2,1, n} h_{2, \alpha^{\prime}}^{\left(\delta^{\prime}\right)}(l) \omega_{1, \alpha-\alpha^{\prime}-1+n}^{\left(\delta-\delta^{\prime}+1-n\right)}(l)+\sum_{n, \alpha^{\prime}, \delta^{\prime}} \mathcal{C}_{\alpha^{\prime}, \alpha-\alpha^{\prime}-2+n}^{2,3, n} h_{2, \alpha^{\prime}}^{\left(\delta^{\prime}\right)}(l) \omega_{3, \alpha-\alpha^{\prime}-2+n}^{\left(\delta-\delta^{\prime}+1-n\right)}{ }^{*}(l), \\
& \partial_{l} \omega_{k \geq 2, \alpha}^{(\delta)}(l)=\sum_{n, \alpha^{\prime}, \delta^{\prime}} \mathcal{D}_{\alpha^{\prime}, \alpha-\alpha^{\prime}+n}^{1, k-1, n}(l) h_{1, \alpha^{\prime}}^{\left(\delta^{\prime}\right)}(l) \omega_{k-1, \alpha-\alpha^{\prime}+n}^{\left(\delta-\delta^{\prime}+1-n\right)}(l)+\sum_{n, \alpha^{\prime}, \delta^{\prime}} \mathcal{C}_{\alpha^{\prime}, \alpha-\alpha^{\prime}-1+n}^{1, k+1, n} h_{1, \alpha^{\prime}}^{\left(\delta^{\prime}\right)}(l) \omega_{k+1, \alpha-\alpha^{\prime}-1+n}^{\left(\delta-\delta^{\prime}+1-n\right)}{ }^{*}(l) \\
& +\sum_{n, \alpha^{\prime}, \delta^{\prime}} \mathcal{D}_{\alpha^{\prime}, \alpha-\alpha^{\prime}+n}^{2, k-2, n} h_{2, \alpha^{\prime}}^{\left(\delta^{\prime}\right)}(l) \omega_{k-2, \alpha-\alpha^{\prime}+n}^{\left(\delta-\delta^{\prime}+1-n\right)}(l)+\sum_{n, \alpha^{\prime}, \delta^{\prime}} \mathcal{C}_{\alpha^{\prime}, \alpha-\alpha^{\prime}-2+n}^{2, k+2, n} h_{2, \alpha^{\prime}}^{\left(\delta^{\prime}\right)}(l) \omega_{k+2, \alpha-\alpha^{\prime}-2+n}^{\left(\delta-\delta^{\prime}+1-n\right)}{ }^{*}(l) .
\end{aligned}
$$

In the above equations, the asterix denotes complex con-

jugation, $\Re$ the real part and we have defined the coeffi- 
cients

$$
\mathcal{D}_{\alpha^{\prime}, \alpha^{\prime \prime}}^{j^{\prime}, j^{\prime \prime}, n}=n !\left(C_{\alpha^{\prime}}^{n} C_{\alpha^{\prime \prime}+j^{\prime \prime}}^{n}-C_{\alpha^{\prime}+j^{\prime}}^{n} C_{\alpha^{\prime \prime}}^{n}\right),
$$

arising from the calculation of the commutator

$$
\left[a^{\dagger^{j^{\prime}}} A_{\alpha^{\prime}}, a^{\dagger j^{\prime \prime}} A_{\alpha^{\prime \prime}}\right]=\sum_{n} \mathcal{D}_{\alpha^{\prime}, \alpha^{\prime \prime}}^{j^{\prime}, j^{\prime \prime}, n} a^{j^{\prime}+j^{\prime \prime}} A_{\alpha^{\prime}+\alpha^{\prime \prime}-n}
$$

The initial conditions for $\tilde{S}_{z}$, for example, are

$$
\omega_{0,0}^{(0)}(l=0)=1 / 2, \quad \omega_{0,1}^{(0)}(l=0)=-1
$$

and all other coefficients are zero. Once the solution of the flow equations for the Hamiltonian are known, the flow equations for the observables can be solved.

\section{APPENDIX B: INTEGRATION OF THE FLOW EQUATIONS}

In this appendix, we explain how to solve the flow equations, order by order in the $1 / N$ expansion. For the observables, we will focus on $\tilde{S}_{z}$, since the computations for $\tilde{S}_{x}$ and $\tilde{S}_{y}$ differ only with respect to the initial conditions (and also the solutions for $\tilde{S}_{z}$ are found to be simpler, which makes this Appendix more readable). Furthermore, $\tilde{S}_{z}(l=0)$ has real coefficients, so this will remain true for any $l$, since $\eta(l)$ also has real coefficients. This allows us to drop the real part $\Re$ in Eqs. (A8)- A10). The same applies to $\tilde{S}_{x}$, but not to $\tilde{S}_{y}$ whose coefficients are purely imaginary.

\section{Order $(1 / N)^{-1},(1 / N)^{-1 / 2}$, and $(1 / N)^{0}$}

\section{a. Flow of the Hamiltonian}

The two first orders for the Hamiltonian have already been discussed in the main text: $h_{0,0}^{(0)}(l)=e_{0}(\infty)$ to order $(1 / N)^{-1}$ and $h_{1,0}^{(0)}(l)=0$ to order $(1 / N)^{-1 / 2}$. Further, we recall how crucial it is to ensure that $h_{1,0}^{(0)}(l=0)=0$, so that this remains true at any $l$. In the following, we will thus drop all contributions to the flow equations involving this term.

To order $(1 / N)^{0}$, the flow equations are given by Eqs. (89)- (91). The equations for $h_{0,1}^{(0)}(l)$ and $h_{2,0}^{(0)}(l)$ are easily solved by noticing that $h_{0,1}^{(0)}(l)^{2}-4 h_{2,0}^{(0)}(l)^{2}$ is a constant of the flow. One gets the hyperbolic solutions

$$
\begin{aligned}
& h_{0,1}^{(0)}(l)=\frac{\Delta^{(\mathrm{R})}}{\tanh \left[2 \Delta^{(\mathrm{R})}\left(l+l_{0}\right)\right]}=\Delta^{(\mathrm{B})} f_{1}(l), \\
& h_{2,0}^{(0)}(l)=\frac{-\operatorname{sgn}(\varepsilon) \Delta^{(\mathrm{R})}}{2 \sinh \left[2 \Delta^{(\mathrm{R})}\left(l+l_{0}\right)\right]}=\Gamma^{(\mathrm{B})} f_{2}(l),
\end{aligned}
$$

where $l_{0}$ is such that the initial conditions are fulfilled, namely $\Delta^{(\mathrm{B})}=\Delta^{(\mathrm{R})} / \tanh \left[2 \Delta^{(\mathrm{R})} l_{0}\right]$. The quantities
$\Delta^{(\mathrm{B})}, \Gamma^{(\mathrm{B})}, \Delta^{(\mathrm{R})}$, and $\varepsilon$ are defined in Sec. IV The functions $f_{1}(l)$ and $f_{2}(l)$ are defined by the two equations above, and satisfy $f_{1}(0)=f_{2}(0)=1$. Finally, $h_{0,0}^{(1)}(l)$ is found thanks to the second constant of the flow, namely $2 h_{0,0}^{(1)}(l)-h_{0,1}^{(0)}(l)$.

There is also another useful representation of the solutions (B1) and (B2). Let us introduce a new "time" scale

$$
t=\operatorname{sgn}(\varepsilon) \exp \left[2 \Delta^{(\mathrm{R})}\left(l+l_{0}\right)\right],
$$

with initial conditions now given at

$$
t_{0}=\operatorname{sgn}(\varepsilon) \exp \left(2 \Delta^{(\mathrm{R})} l_{0}\right)
$$

After some algebra $t_{0}$ can also be shown to be equal to

$$
t_{0}=\frac{1}{\varepsilon}\left(1+\sqrt{1-\varepsilon^{2}}\right) .
$$

Equations (B1) and B2 now read

$$
\begin{aligned}
h_{0,1}^{(0)}(t) & =\Delta^{(\mathrm{R})} \frac{t^{2}+1}{t^{2}-1}, \\
h_{2,0}^{(0)}(t) & =-\Delta^{(\mathrm{R})} \frac{t}{t^{2}-1} .
\end{aligned}
$$

The renormalized values at $l \rightarrow \infty$ are now found by taking the limit $t \rightarrow t_{\infty}=\operatorname{sgn}(\varepsilon) \infty$. Let us remark that the off-diagonal coupling $h_{2,0}^{(0)}(t)$ goes to zero and behaves as $t^{-1}$ for $t \rightarrow t_{\infty}$. This will be true for all off-diagonal couplings creating two excitations since the energy cost of such excitations, in the thermodynamic limit and for large $t$, is nothing but $2 \Delta^{(\mathrm{R})}$, so that the couplings must vanish as $\exp \left(-2 \Delta^{(\mathrm{R})} l\right)$.

\section{b. Flow of the $\tilde{S}_{z}$ observable}

To order $(1 / N)^{-1}$ the flow is

$$
\partial_{l} \omega_{0,0}^{(0)}(l)=0
$$

As a consequence, $\omega_{0,0}^{(0)}(l)=\omega_{0,0}^{(0)}(0)=1 / 2$.

To order $(1 / N)^{-1 / 2}$, there is only one flow equation, namely

$$
\partial_{l} \omega_{1,0}^{(0)}(l)=-2 h_{2,0}^{(0)}(l) \omega_{1,0}^{(0)}(l),
$$

Since for $\tilde{S}_{z}$ the initial condition is $\omega_{1,0}^{(0)}(0)=0$, one gets $\omega_{1,0}^{(0)}(l)=0$.

Working to order $(1 / N)^{0}$ one has three flow equations to solve, which are

$$
\begin{aligned}
& \partial_{l} \omega_{0,0}^{(1)}(l)=-2 h_{1,0}^{(1)} \omega_{1,0}^{(0)}(l)-4 h_{2,0}^{(0)}(l) \omega_{2,0}^{(0)}(l) \\
& \partial_{l} \omega_{0,1}^{(0)}(l)=-4 h_{1,1}^{(0)} \omega_{1,0}^{(0)}(l)-8 h_{2,0}^{(0)}(l) \omega_{2,0}^{(0)}(l) \\
& \partial_{l} \omega_{2,0}^{(0)}(l)=h_{1,0}^{(1)} \omega_{1,0}^{(0)}(l)-2 h_{2,0}^{(0)}(l) \omega_{0,1}^{(0)}(l)
\end{aligned}
$$


For the $\tilde{S}_{z}$ observable considered here for illustration, the previous order solution tells us that the first terms in the three right-hand sides (RHSs) of the above equations vanish, so that the equations are homogeneous. Let us stress that it is not the case for $\tilde{S}_{x}$. One first has to solve the subsystem (B11) and (B12), and then the RHS of (B10) is known so that it can also be integrated. In the $t$ parametrization, one has

$$
\begin{aligned}
\partial_{t} \omega_{0,0}^{(1)}(t) & =-\frac{2}{1-t^{2}} \omega_{2,0}^{(0)}(t), \\
\partial_{t} \omega_{0,1}^{(0)}(t) & =-\frac{4}{1-t^{2}} \omega_{2,0}^{(0)}(t), \\
\partial_{t} \omega_{2,0}^{(0)}(t) & =-\frac{1}{1-t^{2}} \omega_{0,1}^{(0)}(t),
\end{aligned}
$$

which is integrated in

$$
\begin{aligned}
\omega_{0,0}^{(1)}(t) & =-\frac{\left(t-t_{0}\right)^{2}}{\left(t^{2}-1\right)\left(t_{0}^{2}-1\right)} \\
\omega_{0,1}^{(0)}(t) & =-\frac{1+t_{0}^{2}-4 t_{0} t+t^{2}+t_{0}^{2} t^{2}}{\left(t^{2}-1\right)\left(t_{0}^{2}-1\right)} \\
\omega_{2,0}^{(0)}(t) & =\frac{\left(t-t_{0}\right)\left(1-t_{0} t\right)}{\left(t^{2}-1\right)\left(t_{0}^{2}-1\right)}
\end{aligned}
$$

satisfying the initial conditions $\omega_{0,0}^{(1)}\left(t_{0}\right)=0, \omega_{0,1}^{(0)}\left(t_{0}\right)=$ -1 and $\omega_{2,0}^{(0)}\left(t_{0}\right)=0$. From these, one can deduce the renormalized values

$$
\omega_{0,0}^{(1)}\left(t_{\infty}\right)=-\frac{1}{t_{0}^{2}-1}=\frac{1}{2}\left(1-\frac{1}{\sqrt{1-\varepsilon^{2}}}\right),
$$

$$
\begin{aligned}
& \omega_{0,1}^{(0)}\left(t_{\infty}\right)=-\frac{t_{0}^{2}+1}{t_{0}^{2}-1}=-\frac{1}{\sqrt{1-\varepsilon^{2}}}, \\
& \omega_{2,0}^{(0)}\left(t_{\infty}\right)=-\frac{t_{0}}{t_{0}^{2}-1}=-\frac{\varepsilon}{2 \sqrt{1-\varepsilon^{2}}} .
\end{aligned}
$$

Up to a factor $1 / 2$, Eq. B19 is nothing but the $1 / N$ term in Eq. (51).

\section{Flow of the Hamiltonian at order $(1 / N)^{1 / 2}$}

The flow equations read

$$
\begin{aligned}
\partial_{l} h_{1,0}^{(1)}(l)= & -\left[h_{0,1}^{(0)}(l)+4 h_{2,0}^{(0)}(l)\right] h_{1,0}^{(1)}(l) \\
& -4 h_{2,0}^{(0)}(l) h_{1,1}^{(0)}(l), \\
\partial_{l} h_{1,1}^{(0)}(l)= & -\left[h_{0,1}^{(0)}(l)+8 h_{2,0}^{(0)}(l)\right] h_{1,1}^{(0)}(l),
\end{aligned}
$$

with $h_{1,1}^{(0)}(0)=2 h_{1,0}^{(1)}(0)=2 m \sqrt{1-m^{2}}$. The second equation is independent of the first one so it can be solved separately. Then its solution can be inserted in the first equation. This first equation is then solved in two steps, the first being to solve the homogeneous equation, and the second being to find a particular solution of the inhomogeneous solution. Again, the simplest way to solve these equations is to use the $t$ timescale. We simply give the solutions, which read

$$
\begin{aligned}
& h_{1,0}^{(1)}(t)=-m \sqrt{1-m^{2}} \frac{t^{1 / 2}(t-1)^{1 / 2}\left(t_{0}+1\right)^{3 / 2}\left(1+3 t_{0}-3 t-t_{0} t\right)}{t_{0}^{1 / 2}\left(t_{0}-1\right)^{3 / 2}(t+1)^{5 / 2}}, \\
& h_{1,1}^{(0)}(t)=2 m \sqrt{1-m^{2}} \frac{t^{1 / 2}(t-1)^{3 / 2}\left(t_{0}+1\right)^{5 / 2}}{t_{0}^{1 / 2}\left(t_{0}-1\right)^{3 / 2}(t+1)^{5 / 2}}
\end{aligned}
$$

We see that both these off-diagonal couplings go to zero and behave as $t^{-1 / 2}$ for $t \rightarrow t_{\infty}$. This is a general feature of all off-diagonal couplings creating one excitation since their energy cost, in the thermodynamic limit and for large $t$, is nothing but $\Delta^{(\mathrm{R})}$, meaning that these couplings have to go to zero as $\exp \left(-\Delta^{(\mathrm{R})} l\right)$.

\section{Next orders}

¿From order $(1 / N)^{1}$ for the Hamiltonian and $(1 / N)^{1 / 2}$ for the observables, things really become more involved, and it does not make any sense any more to give the solutions in this Appendix. However, we will explain how one can find these solutions.
Stein gave some explicit solutions for the flow of the Hamiltonian in the symmetric phase and in the $l$ timescale language $\left[\text { up to order }(1 / N)^{1}\right]^{47}$. The solutions he gives are found to be polynomials in three functions: $f_{1}(l), f_{2}(l)$ [see Eqs. (B1) and (B2)] and $f_{3}(l)=l+1$ [satisfying the same initial condition as $f_{1}$ and $f_{2}$, namely, $\left.f_{3}(0)=1\right]$. The degree of the polynom in $f_{1}$ can be restricted to be one, since one has $f_{1}^{2}(l)-\varepsilon^{2} f_{2}^{2}(l)=1-\varepsilon^{2}$. In Stein's solutions, the exponential decrease of all offdiagonal couplings creating two excitations is found to mean that one can factor out a term $f_{2}(l)$.

We have checked that to the maximal orders at which we worked, an ansatz of the following form:

$$
h_{0, \alpha}^{(\delta)}(l)=P_{0, \alpha}^{(\delta)}\left[f_{1}(l), f_{2}(l), f_{3}(l)\right]
$$




$$
\begin{aligned}
& h_{1, \alpha}^{(\delta)}(l)=f_{2}^{1 / 2}(l) P_{1, \alpha}^{(\delta)}\left[f_{1}(l), f_{2}(l), f_{3}(l)\right] \\
& h_{2, \alpha}^{(\delta)}(l)=f_{2}(l) P_{2, \alpha}^{(\delta)}\left[f_{1}(l), f_{2}(l), f_{3}(l)\right]
\end{aligned}
$$

is always a solution provided the degrees in $f_{2}$ and $f_{3}$ of the polynoms $P_{0}, P_{1}$, and $P_{2}$ are large enough. The prefactor $f_{2}^{1 / 2}(l)$ in the solution for $h_{1}$ ensures the exponential decrease of these terms creating one excitation, with a time-scale being half the one for terms creating two excitations.

In the $t$ time-scale language, $f_{2}(l)$ reads

$$
f_{2}(l)=\frac{t\left(t_{0}^{2}-1\right)}{t_{0}\left(t^{2}-1\right)} \text {. }
$$

The $P$ polynomial functions translate in fractions having the following properties. The denominator is of the form $(t-1)^{n_{-}}(t-1)^{n_{+}}$, with $n_{-}$and $n_{+}$being integers. The numerator is a polynomial function in $t$ and in $\ln \left(t / t_{0}\right)$ (this logarithm is $f_{3}$ up to multiplicative constant).

\section{APPENDIX C: COEFFICIENTS OF THE $1 / N$ EXPANSION}

In this appendix, we give the first terms of the $1 / N$ expansion for various quantities. Note that we could have only given $\langle H\rangle / N=e_{0}(N),\left\langle S_{z}\right\rangle$, and $\left\langle S_{z}^{2}\right\rangle$ which are sufficient to compute all these quantities (except the gap). Indeed, one has

$$
\langle H\rangle=-\frac{2 \lambda}{N}\left(\left\langle S_{x}^{2}\right\rangle+\gamma\left\langle S_{y}^{2}\right\rangle\right)-2 h\left\langle S_{z}\right\rangle+\frac{\lambda}{2}(1+\gamma)
$$

and

$$
\left\langle\mathbf{S}^{2}\right\rangle=\left\langle S_{x}^{2}\right\rangle+\left\langle S_{y}^{2}\right\rangle+\left\langle S_{z}^{2}\right\rangle=\frac{N}{2}\left(\frac{N}{2}+1\right) .
$$

However, we have considered that explicit forms of $\left\langle S_{x}^{2}\right\rangle,\left\langle S_{y}^{2}\right\rangle$ are of interest because of subtle cancellations of some coefficients.

For the special case $\gamma=-1$, we emphasize that CUTs have already been used by several groups $52,53.54$, notably to compute the $1 / N$ corrections 47 . In addition, these corrections have also been obtained by a perturbative method 55 .

\section{Symmetric phase}

In what follows, we denote by $\Xi(h, \gamma)=(h-1)(h-\gamma)$.

a. Ground-state energy per spin $e_{0}(N)$

$$
\begin{aligned}
e_{0}(N)= & -h+\frac{1}{N}\left[\Xi(h, \gamma)^{1 / 2}+\frac{1}{2}(1+\gamma)-h\right]+\frac{1}{N^{2}}\left[\frac{P_{e}^{(1)}(h, \gamma)}{\Xi(h, \gamma)}+\frac{Q_{e}^{(1)}(h, \gamma)}{\Xi(h, \gamma)^{1 / 2}}\right] \\
& +(1-\gamma)^{2}\left\{\frac{1}{N^{3}}\left[\frac{P_{e}^{(2)}(h, \gamma)}{\Xi(h, \gamma)^{5 / 2}}+\frac{Q_{e}^{(2)}(h, \gamma)}{\Xi(h, \gamma)^{2}}\right]+\frac{1}{N^{4}}\left[\frac{P_{e}^{(3)}(h, \gamma)}{\Xi(h, \gamma)^{4}}+\frac{Q_{e}^{(3)}(h, \gamma)}{\Xi(h, \gamma)^{7 / 2}}\right]\right\}+O\left(\frac{1}{N^{5}}\right)
\end{aligned}
$$

where the $P$ 's and $Q$ 's are polynomial functions of $h$ and $\gamma$ that we list below

$$
\begin{aligned}
& P_{e}^{(1)}(h, \gamma)=\frac{1}{2}(1+\gamma)\left(h^{2}+\gamma\right)-\frac{1}{8} h\left(1+14 \gamma+\gamma^{2}\right) \\
& Q_{e}^{(1)}(h, \gamma)=-\frac{1}{2}(1+\gamma) h+\gamma \\
& P_{e}^{(2)}(h, \gamma)=\frac{1}{16}(1+\gamma) h\left(h^{2}-\gamma\right)-\frac{1}{64}\left[24 h^{4}+\left(1-42 \gamma+\gamma^{2}\right) h^{2}+16 \gamma^{2}\right], \\
& Q_{e}^{(2)}(h, \gamma)=\frac{3}{8} h\left(h^{2}-\gamma\right),
\end{aligned}
$$




$$
\begin{aligned}
P_{e}^{(3)}(h, \gamma)= & \frac{3}{128}(1+\gamma)\left[32 h^{6}+\left(1+14 \gamma+\gamma^{2}\right) h^{4}+\gamma\left(1-66 \gamma+\gamma^{2}\right) h^{2}+16 \gamma^{3}\right] \\
& -\frac{3}{512} h\left[20\left(1+30 \gamma+\gamma^{2}\right) h^{4}+\left(1+36 \gamma-842 \gamma^{2}+36 \gamma^{3}+\gamma^{4}\right) h^{2}-4 \gamma^{2}\left(11-54 \gamma+11 \gamma^{2}\right)\right], \\
Q_{e}^{(3)}(h, \gamma)= & -\frac{1}{128}(1+\gamma) h\left[96 h^{4}+\left(1-34 \gamma+\gamma^{2}\right) h^{2}-64 \gamma^{2}\right] \\
& -\frac{1}{64}\left[\left(4-184 \gamma+4 \gamma^{2}\right) h^{4}-\gamma\left(5-202 \gamma+5 \gamma^{2}\right) h^{2}-16 \gamma^{3}\right] .
\end{aligned}
$$

\section{b. Gap $\Delta(N)$}

$$
\begin{aligned}
\Delta(N)= & 2 \Xi(h, \gamma)^{1 / 2}+\frac{1}{N}\left[\frac{P_{\Delta}^{(1)}(h, \gamma)}{\Xi(h, \gamma)}+\frac{Q_{\Delta}^{(1)}(h, \gamma)}{\Xi(h, \gamma)^{1 / 2}}\right] \\
& +(1-\gamma)^{2}\left\{\frac{1}{N^{2}}\left[\frac{P_{\Delta}^{(2)}(h, \gamma)}{\Xi(h, \gamma)^{5 / 2}}+\frac{Q_{\Delta}^{(2)}(h, \gamma)}{\Xi(h, \gamma)^{2}}\right]+\frac{1}{N^{3}}\left[\frac{P_{\Delta}^{(3)}(h, \gamma)}{\Xi(h, \gamma)^{4}}+\frac{Q_{\Delta}^{(3)}(h, \gamma)}{\Xi(h, \gamma)^{7 / 2}}\right]\right\}+O\left(\frac{1}{N^{4}}\right)
\end{aligned}
$$

with

$$
\begin{aligned}
P_{\Delta}^{(1)}(h, \gamma)= & 2(1+\gamma)\left(h^{2}+\gamma\right)-\frac{1}{2} h\left(1+14 \gamma+\gamma^{2}\right) \\
Q_{\Delta}^{(1)}(h, \gamma)= & -(1+\gamma) h+2 \gamma \\
P_{\Delta}^{(2)}(h, \gamma)= & \frac{1}{8}(1+\gamma) h\left(h^{2}-\gamma\right)-\frac{1}{8}\left[18 h^{4}+\left(1-36 \gamma+\gamma^{2}\right) h^{2}+16 \gamma^{2}\right] \\
Q_{\Delta}^{(2)}(h, \gamma)= & \frac{3}{2} h\left(h^{2}-\gamma\right) \\
P_{\Delta}^{(3)}(h, \gamma)= & \frac{3}{64}(1+\gamma)\left[144 h^{6}+\left(7-62 \gamma+7 \gamma^{2}\right) h^{4}+\gamma\left(7-222 \gamma+7 \gamma^{2}\right) h^{2}+112 \gamma^{3}\right] \\
& -\frac{3}{256} h\left[2560 \gamma h^{4}+\left(7+212 \gamma-4534 \gamma^{2}+212 \gamma^{3}+7 \gamma^{4}\right) h^{2}-128 \gamma^{2}\left(1-14 \gamma+\gamma^{2}\right)\right] \\
Q_{\Delta}^{(3)}(h, \gamma)= & -\frac{1}{16}(1+\gamma) h\left[84 h^{4}+\left(1-34 \gamma+\gamma^{2}\right) h^{2}-52 \gamma^{2}\right] \\
& -\frac{1}{16}\left[\left(5-314 \gamma+5 \gamma^{2}\right) h^{4}-7 \gamma\left(1-50 \gamma+\gamma^{2}\right) h^{2}-32 \gamma^{3}\right]
\end{aligned}
$$

c. One-spin expectation value $\left\langle S_{z}\right\rangle$

$$
\begin{aligned}
\frac{2\left\langle S_{z}\right\rangle}{N}= & 1+\frac{1}{N}\left[\frac{P_{z}^{(1)}(h, \gamma)}{\Xi(h, \gamma)^{1 / 2}}+1\right] \\
& +(1-\gamma)^{2}\left\{\frac{1}{N^{2}}\left[\frac{P_{z}^{(2)}(h, \gamma)}{\Xi(h, \gamma)^{2}}+\frac{Q_{z}^{(2)}(h, \gamma)}{\Xi(h, \gamma)^{3 / 2}}\right]+\frac{1}{N^{3}}\left[\frac{P_{z}^{(3)}(h, \gamma)}{\Xi(h, \gamma)^{7 / 2}}+\frac{Q_{z}^{(3)}(h, \gamma)}{\Xi(h, \gamma)^{3}}\right]\right\}+O\left(\frac{1}{N^{4}}\right),
\end{aligned}
$$

with

$$
\begin{aligned}
P_{z}^{(1)}(h, \gamma) & =\frac{1}{2}(1+\gamma)-h \\
P_{z}^{(2)}(h, \gamma) & =\frac{3}{8}\left(h^{2}-\gamma\right) \\
Q_{z}^{(2)}(h, \gamma) & =-\frac{1}{4} h \\
P_{z}^{(3)}(h, \gamma) & =-\frac{1}{128}(1+\gamma)\left[56 h^{4}-\left(1-98 \gamma+\gamma^{2}\right) h^{2}-88 \gamma^{2}\right]
\end{aligned}
$$




$$
\begin{aligned}
& -\frac{1}{64} h\left[24 h^{4}+\left(1-226 \gamma+\gamma^{2}\right) h^{2}-8 \gamma\left(1-19 \gamma+\gamma^{2}\right)\right], \\
Q_{z}^{(3)}(h, \gamma)= & \frac{3}{8}(1+\gamma) h\left(h^{2}+\gamma\right)+\frac{3}{8}\left(h^{4}-6 \gamma h^{2}+\gamma^{2}\right) .
\end{aligned}
$$

d. Two-spin expectation value $\left\langle S_{x}^{2}\right\rangle$

$$
\begin{aligned}
\frac{4\left\langle S_{x}^{2}\right\rangle}{N^{2}}= & (h-\gamma)\left\{\frac{1}{N} \frac{1}{\Xi(h, \gamma)^{1 / 2}}+\frac{1}{N^{2}}\left[\frac{P_{x x}^{(2)}(h, \gamma)}{\Xi(h, \gamma)^{2}}+\frac{Q_{x x}^{(2)}(h, \gamma)}{\Xi(h, \gamma)^{3 / 2}}\right]\right. \\
& \left.+\frac{1}{N^{3}}\left[\frac{P_{x x}^{(3)}(h, \gamma)}{\Xi(h, \gamma)^{7 / 2}}+\frac{Q_{x x}^{(3)}(h, \gamma)}{\Xi(h, \gamma)^{3}}\right]\right\}+O\left(\frac{1}{N^{4}}\right),
\end{aligned}
$$

with

$$
\begin{aligned}
P_{x x}^{(2)}(h, \gamma)= & -\frac{1}{4}\left[4 h^{3}-2(1+5 \gamma) h^{2}+\left(1+8 \gamma+3 \gamma^{2}\right) h-4 \gamma\right], \\
Q_{x x}^{(2)}(h, \gamma)= & \frac{1}{2}\left[2 h^{2}-(1+3 \gamma) h+2 \gamma\right] \\
P_{x x}^{(3)}(h, \gamma)= & \frac{1}{64}\left[96(1-\gamma) h^{5}-128 \gamma(1-\gamma) h^{4}+4\left(3-65 \gamma+89 \gamma^{2}-27 \gamma^{3}\right) h^{3}\right. \\
& \left.-\left(3-76 \gamma-62 \gamma^{2}+148 \gamma^{3}-13 \gamma^{4}\right) h^{2}-4 \gamma\left(3-33 \gamma+49 \gamma^{2}-19 \gamma^{2}\right) h-16 \gamma^{2}\left(3-2 \gamma-\gamma^{2}\right)\right], \\
Q_{x x}^{(3)}(h, \gamma)= & -\frac{3}{4}(1-\gamma) h\left[2 h^{3}-2 \gamma h^{2}-\gamma(3-\gamma) h+\gamma(1+\gamma)\right] .
\end{aligned}
$$

e. Two-spin expectation value $\left\langle S_{y}^{2}\right\rangle$

$$
\begin{aligned}
\frac{4\left\langle S_{y}^{2}\right\rangle}{N^{2}}= & \frac{1}{h-\gamma}\left\{\frac{1}{N} \Xi(h, \gamma)^{1 / 2}+\frac{1}{N^{2}}\left[\frac{P_{y y}^{(2)}(h, \gamma)}{\Xi(h, \gamma)}+\frac{Q_{y y}^{(2)}(h, \gamma)}{\Xi(h, \gamma)^{1 / 2}}\right]\right. \\
& \left.+\frac{1}{N^{3}}\left[\frac{P_{y y}^{(3)}(h, \gamma)}{\Xi(h, \gamma)^{5 / 2}}+\frac{Q_{y y}^{(3)}(h, \gamma)}{\Xi(h, \gamma)^{2}}\right]\right\}+O\left(\frac{1}{N^{4}}\right),
\end{aligned}
$$

with

$$
\begin{aligned}
P_{y y}^{(2)}(h, \gamma)= & -\frac{1}{4}\left[4 h^{3}-2(5+\gamma) h^{2}+\left(3+8 \gamma+\gamma^{2}\right) h-4 \gamma^{2}\right], \\
Q_{y y}^{(2)}(h, \gamma)= & \frac{1}{2}\left[2 h^{2}-(3+\gamma) h+2 \gamma\right], \\
P_{y y}^{(3)}(h, \gamma)= & -\frac{1}{64}\left[96(1-\gamma) h^{5}-128(1-\gamma) h^{4}+4\left(27-89 \gamma+65 \gamma^{2}-3 \gamma^{3}\right) h^{3}\right. \\
& \left.-\left(13-148 \gamma+62 \gamma^{2}+76 \gamma^{3}-3 \gamma^{4}\right) h^{2}-4 \gamma\left(19-49 \gamma+33 \gamma^{2}-3 \gamma^{2}\right) h-16 \gamma^{2}\left(1+2 \gamma-3 \gamma^{2}\right)\right], \\
Q_{y y}^{(3)}(h, \gamma)= & \frac{3}{4}(1-\gamma) h\left[2 h^{3}-2 h^{2}+(1-3 \gamma) h+\gamma(1+\gamma)\right] .
\end{aligned}
$$

\section{f. Two-spin expectation value $\left\langle S_{z}^{2}\right\rangle$}

$$
\frac{4\left\langle S_{z}^{2}\right\rangle}{N^{2}}=1+\frac{1}{N}\left[\frac{P_{z z}^{(1)}(h, \gamma)}{\Xi(h, \gamma)^{1 / 2}}+2\right]+\frac{1}{N^{2}}\left[\frac{P_{z z}^{(2)}(h, \gamma)}{\Xi(h, \gamma)^{2}}+\frac{Q_{z z}^{(2)}(h, \gamma)}{\Xi(h, \gamma)^{3 / 2}}\right]
$$




$$
+\frac{1}{N^{3}}(1-\gamma)^{2}\left[\frac{P_{z z}^{(3)}(h, \gamma)}{\Xi(h, \gamma)^{7 / 2}}+\frac{Q_{z z}^{(3)}(h, \gamma)}{\Xi(h, \gamma)^{3}}\right]+O\left(\frac{1}{N^{4}}\right),
$$

with

$$
\begin{aligned}
P_{z z}^{(1)}(h, \gamma)= & (1+\gamma)-2 h=2 P_{z}^{(1)}(h, \gamma), \\
P_{z z}^{(2)}(h, \gamma)= & -\frac{1}{4}(1+\gamma) h\left[16 h^{2}+(1+3 \gamma)(3+\gamma)\right]+\frac{1}{2}\left[4 h^{4}+\left(7+10 \gamma+7 \gamma^{2}\right) h^{2}+4 \gamma^{2}\right], \\
Q_{z z}^{(2)}(h, \gamma)= & (1+\gamma)\left(3 h^{2}+\gamma\right)-\frac{1}{2} h\left[4 h^{2}+3(1+\gamma)^{2}\right], \\
P_{z z}^{(3)}(h, \gamma)= & \frac{1}{64}(1+\gamma)\left[224 h^{4}+\left(13-202 \gamma+13 \gamma^{2}\right) h^{2}+16 \gamma^{2}\right] \\
& -\frac{1}{32} h\left[112 h^{4}+\left(59-102 \gamma+59 \gamma^{2}\right) h^{2}-2 \gamma\left(19-6 \gamma+19 \gamma^{2}\right)\right], \\
Q_{z z}^{(3)}(h, \gamma)= & -\frac{3}{4}(1+\gamma) h\left(3 h^{2}-\gamma\right)+\frac{3}{4} h^{2}\left[4 h^{2}+(1-\gamma)^{2}\right] .
\end{aligned}
$$

\section{Broken phase}

In what follows, we will denote $\Psi(h)=1-h^{2}$.

a. Ground-state energy per spin $e_{0}(N)$

$e_{0}(N)=-\frac{1}{2}\left(1+h^{2}\right)+\frac{1}{N}\left[(1-\gamma)^{1 / 2} \Psi(h)^{1 / 2}-\frac{1}{2}(1-\gamma)\right]+\frac{1}{N^{2}}\left[\frac{\gamma h^{2}-2+\gamma}{2 \Psi(h)}+\frac{(1-\gamma)^{1 / 2}}{\Psi(h)^{1 / 2}}\right]+O\left(\frac{1}{N^{3}}\right)$.

b. $\operatorname{Gap} \Delta(N)$

$\Delta(N)=2(1-\gamma)^{1 / 2} \Psi(h)^{1 / 2}+\frac{1}{N}\left[-2 \frac{(1-2 \gamma) h^{2}+2-\gamma}{\Psi(h)}+\frac{2(1-\gamma)^{1 / 2}}{\Psi(h)^{1 / 2}}\right]+O\left(\frac{1}{N^{2}}\right)$

c. One-spin expectation values

$$
\begin{aligned}
& \frac{2\left\langle S_{x}\right\rangle}{N}=\Psi(h)^{1 / 2}+\frac{1}{N}\left[-\frac{(1-2 \gamma) h^{2}+2-\gamma}{2(1-\gamma)^{1 / 2} \Psi(h)}+\frac{h^{2}}{\Psi(h)^{1 / 2}}+\Psi(h)^{1 / 2}\right]+O\left(\frac{1}{N^{2}}\right), \\
& \frac{2\left\langle S_{y}\right\rangle}{N}=0+O\left(\frac{1}{N^{2}}\right), \\
& \frac{2\left\langle S_{z}\right\rangle}{N}=h+\frac{1}{N}\left[\frac{(1-\gamma)^{1 / 2} h}{\Psi(h)^{1 / 2}}\right]+O\left(\frac{1}{N^{2}}\right) .
\end{aligned}
$$

d. Two-spin expectation values

$$
\frac{4\left\langle S_{x}^{2}\right\rangle}{N^{2}}=\Psi(h)+\frac{1}{N}\left[\frac{h^{2}\left(h^{2}-3+2 \gamma\right)}{(1-\gamma)^{1 / 2} \Psi(h)^{1 / 2}}+2+\frac{\left(h^{2}-2+\gamma\right) \Psi(h)^{1 / 2}}{(1-\gamma)^{1 / 2}}\right]+O\left(\frac{1}{N^{2}}\right)
$$




$$
\begin{aligned}
& \frac{4\left\langle S_{y}^{2}\right\rangle}{N^{2}}=\frac{1}{N} \frac{\Psi(h)^{1 / 2}}{(1-\gamma)^{1 / 2}}+O\left(\frac{1}{N^{2}}\right), \\
& \frac{4\left\langle S_{z}^{2}\right\rangle}{N^{2}}=h^{2}+\frac{1}{N}\left[\frac{2(1-\gamma)^{1 / 2} h^{2}}{\Psi(h)^{1 / 2}}+(1-\gamma)^{1 / 2} \Psi(h)^{1 / 2}\right]+O\left(\frac{1}{N^{2}}\right) .
\end{aligned}
$$

* Electronic address: sdusuel@thp.uni-koeln.de

$\dagger$ Electronic address: vidal@lptmc.jussieu.fr

1 A. Osterloh, L. Amico, G. Falci, and R. Fazio, Nature (London) 416, 608 (2002).

2 T. J. Osborne and M. A. Nielsen, Phys. Rev. A 66, 032110 (2002).

3 I. Bose and E. Chattopadhyay, Phys. Rev. A 66, 062320 (2002).

4 F. C. Alcaraz, A. Saguia, and M. S. Sarandy, Phys. Rev. A 70, 032333 (2004).

${ }^{5}$ O. Osenda, Z. Huang, and S. Kais, Phys. Rev. A 67, 062321 (2003).

${ }^{6}$ O. F. Syljuasen, Phys. Rev. A 68, 060301 (2003).

7 O. F. Syljuasen, Phys. Lett. A 322, 25 (2004).

8 U. Glaser, H. Büttner, and H. Fehske, Phys. Rev. A 68, 032318 (2003).

9 S. J. Gu, H. Q. Lin, and Y. Q. Li, Phys. Rev. A 68, 042330 (2003).

10 T. Stauber and F. Guinea, Phys. Rev. A 70, 22313 (2004).

11 F. Verstraete, M. Popp, and J. I. Cirac, Phys. Rev. Lett. 92, 027901 (2004).

12 F. Verstraete, M. A. Martin-Delgado, and J. I. Cirac, Phys. Rev. Lett. 92, 087201 (2004).

13 P. Stelmachovic and V. Buzek, Phys. Rev. A 70, 032313 (2004).

14 C. Wellard and R. Orus, Phys. Rev. A 70, 062318 (2004).

15 J. Reslen, L. Quiroga, and N. F. Johnson, Europhys. Lett. 69, 8 (2005).

16 N. Lambert, C. Emary, and T. Brandes, Phys. Rev. Lett. 92, 073602 (2004).

17 N. Lambert, C. Emary, and T. Brandes, Phys. Rev. A 71, 053804 (2005).

18 G. Vidal, J. I. Latorre, E. Rico, and A. Kitaev, Phys. Rev. Lett. 90, 227902 (2003).

19 J. I. Latorre, E. Rico, and G. Vidal, Quantum Inf. Comput. 4, 48 (2004).

20 B. Q. Jin and V. E. Korepin, Phys. Rev. A 69, 062314 (2004).

21 J. I. Latorre, R. Orús, E. Rico, and J. Vidal, condmat/0409611.

22 V. E. Korepin, Phys. Rev. Lett. 92, 096402 (2004).

${ }^{23}$ H. Fan, V. Korepin, and V. Roychowdhury, Phys. Rev. Lett. 93, 227203 (2004).

24 A. R. Its, B. Q. Jin, and V. E. Korepin, J. Phys. A 38, 2975 (2005).

25 H. J. Lipkin, N. Meshkov, and A. J. Glick, Nucl. Phys. 62, 188 (1965).
26 N. Meshkov, A. J. Glick, and H. J. Lipkin, Nucl. Phys. 62, 199 (1965).

27 A. J. Glick, H. J. Lipkin, and N. Meshkov, Nucl. Phys. 62, 211 (1965).

28 J. I. Cirac, M. Lewenstein, K. Mølmer, and P. Zoller, Phys. Rev. A 57, 1208 (1998).

29 R. Botet, R. Jullien, and P. Pfeuty, Phys. Rev. Lett. 49, 478 (1982).

30 R. Botet and R. Jullien, Phys. Rev. B 28, 3955 (1983).

31 J. Vidal, G. Palacios, and R. Mosseri, Phys. Rev. A 69, 022107 (2004).

32 J. Vidal, R. Mosseri, and J. Dukelsky, Phys. Rev. A 69, 054101 (2004).

33 J. Vidal, G. Palacios, and C. Aslangul, Phys. Rev. A 70, 062304 (2004).

34 S. Dusuel and J. Vidal, Phys. Rev. Lett. 93, 237204 (2004).

${ }^{35}$ F. Pan and J. P. Draayer, Phys. Lett. B 451, 1 (1999).

36 J. Links, H.-Q. Zhou, R. H. McKenzie, and M. D. Gould, J. Phys. A 36, R63 (2003).

37 F. Wegner, Ann. Physik (Leipzig) 3, 77 (1994).

38 S. D. Głazek and K. G. Wilson, Phys. Rev. D 48, 5863 (1993).

39 S. D. Głazek and K. G. Wilson, Phys. Rev. D 49, 4214 (1994)

40 C. M. Newman and L. S. Schulman, J. Math. Phys. 18, 23 (1977).

41 T. Holstein and H. Primakoff, Phys. Rev. 58, 1098 (1940).

42 A. Auerbach, Interacting Electrons and Quantum Magnetism (Springer-Verlag, New-York, 1994).

43 C. Emary and T. Brandes, Phys. Rev. E 67, 066203 (2003).

44 S. Dusuel and G. S. Uhrig, J. Phys. A 37, 9275 (2004).

45 A. Mielke, Eur. Phys. J. B 5, 605 (1998).

46 C. Knetter and G. S. Uhrig, Eur. Phys. J. B 13, 209 (2000).

47 J. Stein, J. Phys. G 26, 377 (2000).

48 W. K. Wootters, Phys. Rev. Lett. 80, 2245 (1998).

49 R. Somma, G. Ortiz, H. Barnum, E. Knill, and L. Viola, Phys. Rev. A 70, 042311 (2004).

50 X. Wang and K. Mølmer, Eur. Phys. J. D 18, 385 (2002).

51 J. von Delft and D. C. Ralph, Phys. Rep. 345, 61 (2001).

${ }^{52}$ H. J. Pirner and B. Friman, Phys. Lett. B 434, 231 (1998).

53 F. G. Scholtz, B. H. Bartlett, and H. B. Geyer, Phys. Rev. Lett. 91, 080602 (2003).

54 J. N. Kriel, A. Y. Morozov, and F. G. Scholtz, J. Phys. A 38, 205 (2005).

55 A. Dzhioev, Z. Aouissat, A. Storozhenko, A. Vdovin, and J. Wambach, Phys. Rev. C 69, 014318 (2004). 